\title{
Three-dimensional binocular eye-hand coordination in normal vision and with simulated visual impairment
}

\author{
Guido Maiello ${ }^{1,2}$, MiYoung Kwon ${ }^{3}$, Peter J. Bex ${ }^{4}$ \\ ${ }^{1}$ UCL Institute of Ophthalmology, University College London, 11-43 Bath Street, London EC1V \\ 9EL, UK \\ 2Present Address: Department of Experimental Psychology, Justus-Liebig University Giessen, \\ Otto-Behaghel-Str.10F, 35394 Giessen, Germany \\ ${ }^{3}$ Department of Ophthalmology, University of Alabama at Birmingham, 700 S. 18th Street, \\ Birmingham, AL 35294-0009, USA \\ ${ }^{4}$ Department of Psychology, Northeastern University, 360 Huntington Ave, Boston, MA 02115, \\ USA
}

\begin{abstract}
Sensorimotor coupling in healthy humans is demonstrated by the higher accuracy of visually tracking intrinsically—rather than extrinsically—generated hand movements in the fronto-parallel plane. It is unknown whether this coupling also facilitates vergence eye movements for tracking objects in depth, or can overcome symmetric or asymmetric binocular visual impairments. Human observers were therefore asked to track with their gaze a target moving horizontally or in depth. The movement of the target was either directly controlled by the observer's hand or followed hand movements executed by the observer in a previous trial. Visual impairments were simulated by blurring stimuli independently in each eye. Accuracy was higher for self-generated movements in all conditions, demonstrating that motor signals are employed by the oculomotor system to improve the accuracy of vergence as well as horizontal eye movements. Asymmetric monocular blur affected horizontal tracking less than symmetric binocular blur, but impaired tracking in depth as much as binocular blur. There was a critical blur level up to which pursuit and vergence eye movements maintained tracking accuracy independent of blur level. Hand-eye coordination may therefore help compensate for functional deficits associated with eye disease and may be employed to augment visual impairment rehabilitation.
\end{abstract}

\section{Keywords}

Smooth pursuit; Vergence; Ocular tracking of self-motion; Eye-hand coordination; Asymmetric visual impairment; Amblyopia and strabismus

Guido Maiello,guido_maiello@yahoo.it.

Author Contributions GM, MK and PJB conceived and designed the study. GM programmed the experiments, and collected and analyzed the data. All authors wrote the manuscript.

Conflict of interest The authors declare that they have no conflict of interest. 


\section{Introduction}

To track moving objects, the visual system executes smooth pursuit eye movements, which hold the high-resolution fovea onto a tracked object by matching its speed (Lisberger 2015). Furthermore, because humans have binocular overlapping visual fields, to inspect objects at different distances or to track objects moving in depth, the oculomotor system also executes vergence eye movements, which are unequal slow rotations of each eye that shift the binocular gaze point in depth (Coubard 2013). Smooth pursuit and vergence eye movements have different tracking characteristics (Semmlow et al. 1998) and rely on separate neural substrates (Gamlin and Yoon 2000; Ward et al. 2015); however, they are similarly encoded in the frontal eye fields region of the frontal cortex (Fukushima et al. 2002; Akao et al. 2005).

To actively interact with the environment, humans not only move their eyes, but also perform a range of 3D hand movements which include reaching, grasping and manipulating objects. To take advantage of correlations that exist between spatial coordinates in visual and action space, the eye and hand motor systems have been found to be linked. For example, Steinbach and Held (1968) showed that smooth pursuit eye movements to self-generated motion are more accurate than to externally generated motion. This finding is consistent with the view that there is an exchange of information between the motor systems of the eyes and hands (Scarchilli and Vercher 1999). Furthermore, Chen et al. (2016) provide convincing electrophysiological evidence that the oculomotor system may receive efference copy from the hand motor system. However, it is also possible that a common command signal controls coordinated hand and smooth pursuit eye movements (Bock 1987).

In contrast to the findings regarding smooth pursuit eye movements, to date no link has been found between hand movements and vergence eye movements in depth. Koken and Erkelens (1993) in particular have shown that simultaneous hand tracking of a target moving sinusoidally in depth does not improve the latency of vergence eye movements executed to visually track the target. This finding is in contrast to previous results by the same authors which demonstrate that simultaneous hand tracking of an externally generated target motion enhances smooth pursuit eye movements (Koken and Erkelens 1992). Thus, the vergence system does not exhibit the same eye-hand coupling as the pursuit system when the eyes and hands are tracking externally generated motion. These findings suggest that the oculomotor system does not employ afferent hand motor signals to enhance vergence tracking. It remains unknown whether vergence eye movements may be enhanced during tracking of internally generated motion in depth. If this were the case, it would provide evidence that either a common command signal controls coordinated hand and vergence eye movements, or that the oculomotor system receives efferent hand motor information when tracking self-generated motion in depth. Thus, the present study aims to investigate whether both pursuit and vergence eye movements may be enhanced during tracking of internally generated motion.

Binocular vision and stereoscopic depth perception are fundamental in everyday eye-hand coordination (Fielder and Moseley 1996; O'Connor et al. 2010; Gonzalez and NiechwiejSzwedo 2016). Consequently, patients with stereovision deficits are known to have 
impairments in reaching and grasping hand movements [e.g., children with amblyopia (Grant et al. 2014; Suttle et al. 2011; Sloper et al. 2011) or adults with age-related macular degeneration (Verghese et al. 2016) or glaucoma (Kotecha et al. 2009)]. One of the most basic and pervasive causes of visual impairment is refractive error (defocus blur), with an estimated 153 million visually impaired people worldwide as a result of uncorrected refractive errors (Resnikoff et al. 2008). Refractive error refers to the inability of the eye to focus light onto the retina. Myopia (nearsightedness), for example, is a common type of refractive error in which the eyes grow too large for the lens system to be able to focus far objects onto the fovea. Refractive error causes retinal images to be blurred and reduces the visibility of small objects and fine spatial detail. No official prevalence data are available, but for different age groups the distribution of myopic and hyperopic refractive errors is not negligible [see Fig. 1 (Sorsby et al. 1960; Hyams et al. 1977; Kamiya 1984; Fan et al. 2004)]. Some visually impaired individuals are defined by the type of refractive error: patients with anisometropic amblyopia tend to have significant hyperopic refractive error that is binocularly asymmetric; consequently, untreated amblyopes have different amounts of blur in the two eyes (Stewart et al. 2004).

Symmetric and asymmetric visual blur may affect smooth pursuit and vergence eye movements in several different ways. Blur reduces both the contrast and the spatial frequency content of visual targets. Spering et al. (2005) have found that reduced contrast impairs pursuit eye movements by affecting the correct estimation of target speed, while reducing spatial frequency has no systematic effect on pursuit eye movements. Conversely, the precision of binocular alignment is known to depend on spatial frequency (Schor et al. 1984; Judge 1996; Maiello et al. 2014). Thus, both smooth pursuit and vergence eye movements are likely to be impaired by symmetric blur in each eye. In conditions of asymmetric blur, unequal contrast and spatial frequency content in the two eyes may lead to suppression of the eye experiencing impoverished visual input (Fahle 1982; Kwon et al. 2015). This interocular suppression is likely to have very different effects on the performance of smooth pursuit and vergence eye movements. During smooth pursuit eye movements, if the input from one eye were suppressed, pursuit performance might still successfully rely on the eye viewing the non-blurred target. Binocular disparity, a necessary component of vergence eye movements, is instead known to be impaired by interocular differences in spatial resolution (Lam et al. 1996). Asymmetric blur is thus likely to impair vergence eye movements, because only the matching low spatial frequency information from the two eyes is available to maintain binocular alignment. Additionally, interocular suppression might mask the remaining matching binocular input altogether, making binocular alignment impossible.

However, it is still poorly understood how eye-hand coupling is affected by reduced target visibility. In conditions of visual uncertainty, the coupling between the eye and hand motor systems could break down. Conversely, von Noorden and Mackensen (1962) showed that humans are able to make smooth pursuit eye movements to their own finger motion in the dark; therefore if the oculomotor system optimally combined visual, hand motor efferent and proprioceptive information, it is possible that eye-hand coupling could be unaffected, or even strengthened, when visual information is unreliable. 
Here, we investigate the coupling between the eye and hand motor systems under conditions of simulated monocular and binocular blur. More specifically, we examine both left-right pursuit eye movements in the fronto-parallel plane as well as vergence eye movements in depth. Because eye-hand coordination occurs in 3D, we ask how eye-hand coupling in depth is affected by blur, and whether monocular and binocular visual impairments have similar effects. We also discuss how our findings from image blur could relate to real-world blur associated with refractive error.

\section{Materials and methods}

\section{Participants}

Five subjects, author GM and four naïve observers, ( 3 male, mean \pm SD age $29 \pm 6$ ) participated in the study. All subjects had normal or corrected to normal vision and normal stereo vision. All subjects reported being right handed and right-eye dominant. All procedures were approved by the Northeastern University Institutional Review Board and adhered to the tenets of the declaration of Helsinki. All subjects provided written informed consent.

\section{Apparatus}

The experiment was programmed with the Psychophysics Toolbox Version 3 (Brainard 1997; Pelli 1997) and Eyelink Toolbox (Cornelissen et al. 2002) in Matlab (MathWorks). Stimuli were presented on an BenQ XL2720Z LCD monitor with a resolution of $1920 \times$ 1080 pixels (display dot pitch $0.311 \mathrm{~mm}$ ) at $120 \mathrm{~Hz}$. The monitor was run from an NVidia Quadro K 420 graphics processing unit. Observers were seated in a dimly lit room, $57 \mathrm{~cm}$ in front of the monitor with their heads stabilized in a chin and forehead rest and wore active wired stereoscopic shutter glasses (NVIDIA 3DVision) during all experiments to control dichoptic stimulus presentation. The cross talk of the dichoptic system was $1 \%$ measured with a Spectrascan 6500 photometer. Eye tracking was performed using the EyeLink 1000 (SR Research) desktop mount eye tracker running at $1000 \mathrm{~Hz}$. The eye tracker was calibrated binocularly using the native five-point calibration routine at the start of each experimental session. Finger tracking was performed using a leap motion controller, a commercial low-cost hand motion tracker recently validated for research applications (Weichert et al. 2013; Guna et al. 2014), which exhibits high accuracy (below $0.2 \mathrm{~mm}$ ), but an inconsistent sampling frequency of $\approx 40 \mathrm{~Hz}$. The leap motion device also exhibits a relatively high end- to-end latency ( $85 \mathrm{~ms}$ ) (Brown et al. 2014) that is nevertheless within a range that does not impair human performance at a range of tasks (Ware and Balakrishnan 1994; Ellis et al. 1997; Teather et al. 2009). Binocular gaze and finger position measurements were queried from the eye and finger tracking devices at the monitor refresh rate of $120 \mathrm{~Hz}$. Missing finger position samples were recovered in real time through secondorder Savitzky-Golay interpolation on the previous 19 samples. The leap motion controller was placed $30 \mathrm{~cm}$ in front and to the right of the observer, so that the observer's right hand could be tracked. The leap motion was calibrated, once for all observers, so that placing the tip of the index finger $20 \mathrm{~cm}$ above the sensor was mapped to the center of the monitor, and left-right, backward and forward finger movements were mapped onto equally sized 
movements from the screen center. Left and right eye on-screen position data recovered from the Eyelink were transformed into the 3D gaze position with respect to the screen center.

\section{Stimuli}

The stimulus for target tracking was a Gabor patch $\left(\sigma=0.25^{\circ}, \omega=2\right.$ cycles $/ \mathrm{deg}, 100 \%$ Michelson contrast) moving on top of a white bar $\left(0.5^{\circ}\right.$ thick and $8^{\circ}$ wide). Stimuli were embedded in 1/f pink noise background which has the same frequency content of natural images (Kretzmer 1952; Bex and Makous 2002) and helped reduce the visibility of stereoscopic cross talk. Example stimuli are shown in Fig. 2. From trial to trial, we systematically varied the amount of blur in the stimulus to one or both eyes using fast Gaussian mipmap filtering. Blur level is specified as double the standard deviation of the Gaussian blur kernel in minutes of arc. In the pursuit sessions, seven blur levels were employed: $[0,6.25,12.5,25,50,100,200]$ arcmin. In the vergence sessions, only the first six blur levels were employed, as in pilot testing the vergence task was found to be already near impossible with 100 arcmin of blur. Note that with increasing blur levels, the contrast of the stimuli decreased. The blur levels employed can be equated to contrast levels of [100, 95, 78, 59, 48, 35, 20]\% Michelson contrast.

In the binocular blur trials, the stimuli presented to both eyes were blurred. In the monocular blur trials, only the stimuli shown to the left eye were blurred, whereas a sharply focused stimulus was always shown to the right eye.

\section{Experimental design}

Observers participated in four experimental sessions on separate days. The four sessions are schematized in Fig. 3. In sessions 1 and 2, which were horizontal pursuit eye movement sessions, observers were required to complete five trials for each of the seven blur levels for both monocular and binocular blur conditions. Thus in sessions 1 and 2, observers completed 70 trials per session. In sessions 3 and 4, which were vergence pursuit eye movement sessions, observers completed five trials for each of the six blur levels for both monocular and binocular blur conditions, for a total of 60 trials per session. Within each session, trial order was fully randomized.

- $\quad$ Session 1: Horizontal pursuit finger tracking (Fig. 3a). At the beginning of each trial, observers placed their index finger above the leap motion controller. When ready, observers pressed, with their left hand, the space bar on the keyboard in front of them to begin the trial recording. Then, observers were required to smoothly move their index finger from right to left and back, directly above the leap motion controller, which tracked the motion of the observer's fingertip. The on-screen Gabor target moved with the observer's finger, and the observer was required to track the Gabor target as accurately as possible with his/her gaze. The motion of the on-screen target was limited to within $\pm 4^{\circ}$ from the center, constrained onto the stimulus bar. Once the observers had completed the movement, they signaled the end of the trial recording by once again lefthandedly pressing the space bar on the keyboard in front of them. If less than $2 \mathrm{~s}$ passed between trial start and finish key presses, the trial was deemed invalid and was repeated. 
- $\quad$ Session 2: Horizontal pursuit replay tracking (Fig. 3b). Observers were required to hold their right hand motionless. When ready, observers pressed, with their left hand, the space bar on the keyboard in front of them to begin each trial recording. Then, the Gabor target would move right to left and back, replaying one of the finger movements executed in session 1, matched to the viewing condition. The observer's task was solely to track the Gabor target as accurately as possible with his/her gaze.

- $\quad$ Session 3: Vergence pursuit finger tracking (Fig. 3c). At the beginning of each trial, observers placed their index finger above the leap motion controller. When ready, observers pressed, with their left hand, the space bar on the keyboard in front of them to begin the trial recording. Then, observers were required to smoothly move their index finger backward and forward, directly above the leap motion controller, which tracked the motion of the observer's fingertip in depth. The on-screen Gabor target moved in depth with the observer's finger, and the observer was required to track (by executing vergence eye movements) the Gabor target as accurately as possible with his/her gaze. The motion of the onscreen dot was limited from 0 to $-8^{\circ}$ of crossed disparity (i.e., the dot could only move from the surface of the screen toward the observer). Once the observers had completed the movement, they signaled the end of trial recording by once again left-handedly pressing the space bar on the keyboard in front of them. If less than $2 \mathrm{~s}$ passed between trial start and finish key presses, the trial was deemed invalid and was repeated.

- $\quad$ Session 4: Vergence pursuit replay tracking (Fig. 3d). Observers were required to hold their right hand motionless. When ready, observers pressed, with their left hand, the space bar on the keyboard in front of them to begin each trial recording. For each trial, the Gabor target moved backward and forward, replaying one of the finger movements in depth executed in session 3, matched to the viewing condition. The observer's task was solely to track the Gabor target as accurately as possible with his/her gaze.

\section{Training}

Prior to sessions 1 and 3, observers performed training to learn the eye-hand coordination task. Prior to session 1, observers performed five practice trials in which they moved their finger right to left and back, and were required to visually track the on-screen Gabor target. Prior to session 2, observers performed five practice trials in which they moved their finger backward and forward, and were required to visually track, via vergence eye movements, the on-screen Gabor target. Observers were informed that if less than $2 \mathrm{~s}$ passed between trial start and finish key presses, the trial would be deemed invalid and have to be repeated. There was no upper time limit on the duration of a trial. In both sets of practice trials, visual stimuli were rendered with no blur to both eyes. Following the practice trials, observers were informed that throughout the main experiment the visibility of the visual stimuli would vary. 


\section{Statistical analyses: a priori, hypothesis-driven analyses}

An initial aim of this study was to test whether both pursuit and vergence eye movements to self-generated hand movements are more accurate than eye movements to externally generated motion. We further tested whether visual blur in one or both eyes disrupts visual tracking left right and in depth. Lastly, we tested whether the link between the eye and hand motor systems could facilitate oculomotor target tracking in conditions of visual uncertainty.

To test our experimental hypotheses, analyses were designed a priori by piloting our experimental design (Maiello et al. 2016). Pilot data showed that by measuring tracking accuracy as the correlation between gaze and target position, we could reliably measure differences in accuracy as small as $1 \%$ for both pursuit and vergence eye movements. These pilot data also showed that the dependency of tracking accuracy as a function of blur was clearly not linear, but could be described by a horizontal asymptote at blur levels near zero and a steep fall-off at high blur levels. On linear-log axes, either a decreasing exponential function or its two-section piecewise linearization, a hinged-line function, described the pilot data equally well with the same number of free parameters. The hinged-line function was chosen over the decreasing exponential function because the fitted parameters lend themselves to a more immediate interpretation of the results, as we explain below. Thus, tracking data from our experiment was analyzed as follows.

In each trial, tracking accuracy was computed as the correlation between gaze position and target position either along the fronto-parallel plane (horizontal pursuit) or the sagittal plane (vergence pursuit). Figure 4 shows the tracking accuracy as a function of blur for a representative observer in the binocular blur, horizontal pursuit finger tracking condition. These data were first transformed via Fisher's $Z$ transformation to ensure variance stabilization (Fisher 1915). Correlation data were then fit to a hinged-line linearlog function with equation:

$$
y=\left\{\begin{array}{ll}
b & : \log _{2}(x)<c \\
d \log _{2}(x)+b-d c & : \log _{2}(x) \geq c
\end{array},\right.
$$

where $b$ is an observer's baseline level of tracking accuracy, $c$ is the critical blur level at which tracking performance begins to deteriorate, and $d$ is the rate of decay of tracking accuracy with increasing levels of blur beyond $c$.

If a link between eye and hand movements exists for both left-right and in-depth tracking, we would expect higher baseline accuracy in the finger tracking condition for both pursuit and vergence eye moments. If blur in one or both eyes impairs left-right and in-depth tracking differently, this would be reflected either by changes in the level of critical blur or by changes in the rate of decay of tracking accuracy. If the link between the eye and hand motor systems were to break down in conditions of visual uncertainty, we would expect either smaller critical blur or steeper decay in the finger tracking condition as compared to the replay tracking condition. Conversely if eye-hand coupling were strengthened in 
conditions of visual uncertainty, we could expect larger critical blur levels or shallower decay rates during finger tracking.

To test these predictions, parameter estimates were analyzed using a 2 (finger vs replay tracking) $\times 2$ (pursuit vs vergence) $\times 2$ (binocular vs monocular blur) within-subject analysis of variance (ANOVA). ANOVA normality assumptions were verified with quantile-quantile plots.

\section{Statistical analyses: a posteriori, exploratory analyses}

To tease apart the mechanisms underlying our primary findings, we proceeded to design the following exploratory analyses of our data. First, we processed the target trajectories of single trials from each experimental condition to obtain the onset of finger/target motion. We initially selected a rough maximum estimate of movement onset, which corresponded to the first position sample in which the target had moved in the direction of motion by at least $0.5^{\circ}$ for horizontal pursuit tracking and by at least $1^{\circ}$ for vergence pursuit tracking. We then searched the movement traces up to this maximum estimate for the true movement onset using the method described by (Schütz et al. 2007; Chen et al. 2016). In short, velocity signals were calculated through digital differentiation, and regression lines with 50-ms length were fitted to these velocity trace. Regression lines with $R^{2}<0.7$ or slope $<0.1$ $\mathrm{deg} / \mathrm{s}^{2}$ were discarded. Of the remaining regression lines, the one with the highest $R^{2}$ value was selected. The intercept of this line with the time axis was defined as the onset of the target motion. All target and eye movement traces from all experimental conditions were then temporally aligned so that time $t=0$ corresponded to the onset of the target motion. We then calculated the duration and mean velocity of the finger movements, as well as the gazetracking error for every trial. Tracking error was computed as the gaze position minus the target position, along the fronto-parallel plane for horizontal pursuit tracking and along the sagittal plane for vergence pursuit tracking.

To quantify the accuracy of open-loop tracking, i.e., tracking at movement onset, for each condition we estimated the onset of eye movements executed to track the target. We first low-pass filtered the eye position data below $10 \mathrm{~Hz}$, then averaged the temporally aligned eye position traces from the five repetitions for each condition. The onset of eye movements was then calculated using the same procedure employed to estimate target movement onset. The latency of horizontal and vergence tracking eye movements was thus defined as the difference between target and eye movement onset, with negative latencies indicating anticipatory eye movements. Latency data in the horizontal pursuit condition were analyzed using a 2 (finger vs replay tracking) $\times 2$ (binocular vs monocular blur) $\times 7$ (blur level) within-subject ANOVA. Latency data in the vergence pursuit condition were analyzed using a 2 (finger vs replay tracking) $\times 2$ (binocular vs monocular blur) $\times 6$ (blur level) withinsubject ANOVA.

To quantify the accuracy of closed-loop tracking, i.e., tracking after movement onset, we instead measured the rate of saccadic eye movements during both horizontal pursuit and vergence tracking. Saccades were detected using a velocity threshold on the horizontal eye position traces of $25 \mathrm{deg} / \mathrm{s}$. Saccade rate was measured as the number of saccades occurring after the target had moved in the direction of motion by at least $0.5^{\circ}$ for horizontal pursuit 
tracking and by at least $1^{\circ}$ for vergence pursuit tracking, divided by the duration of the trial. For horizontal pursuit tracking we included only catch-up saccades, i.e., saccades occurring in the same direction as the target motion. For vergence tracking, we included all saccades occurring during the trial. Saccade rate data in the horizontal pursuit condition were analyzed using a 2 (finger vs replay tracking) $\times 2$ (binocular vs monocular blur) $\times 7$ (blur level) within-subject ANOVA. Saccade rate data in the vergence pursuit condition were analyzed using a 2 (finger vs replay tracking) $\times 2$ (binocular vs monocular blur) $\times 6$ (blur level) within-subject ANOVA.

\section{Results}

\section{Primary findings from hypothesis-driven analyses}

Tracking accuracy varied lawfully as a function of blur level (Fig. 5a,b); accuracy was constant baseline up to a critical blur level, after which accuracy fell off on linear-log axes with a linear rate of decay. This pattern held true for both horizontal pursuit eye movements (Fig. 5a, b squares) and vergence pursuit eye movements (Fig. 5a, b triangles).

3D tracking of internally generated motion is more accurate than tracking of externally generated motion-Tracking accuracy was noticeably higher when observers tracked a stimulus controlled by their own finger movements in real time (black curves, Fig. $5 \mathrm{a}, \mathrm{b})$ than when observers tracked a replay of their own previously executed finger movements (red curves, Fig. 5a, b). Specifically, Fig. 6a shows that baseline accuracy was significantly greater in the finger (black) than the replay (red) tracking condition (Finger vs replay main effect: $F_{1,4}=19.96, p=0.011$ ). Figure 6 a also shows that horizontal pursuit (squares) tracking accuracy was threefold greater (in $Z$-scaled space) than vergence pursuit (triangles) tracking accuracy (horizontal vs vergence main effect on baseline accuracy: $F_{1,4}$ $=153.82, p=0.00024)$. However, the difference between finger and replay conditions was similarly large across horizontal (Cohen's $d=1.37$ ) and in depth tracking (Cohen's $d=0.94$ ) conditions (interaction effect on baseline accuracy between finger vs replay and horizontal vs vergence: $\left.F_{1,4}=0.082, p=0.79\right)$. This result further confirms the coupling between the oculomotor and hand motor systems and shows that the eye and hand motor systems are also linked for movements in 3D space. Sensibly, baseline accuracy did not vary as a function of monocular or binocular blur conditions, as these conditions are the same at baseline for the least blurred condition (monocular vs binocular blur main effect on baseline accuracy: $F_{1,4}=$ $0.078, p=0.79$; all two- and three-way interactions: $p>0.16$ ).

Eye-hand coupling is unaffected by blur-Tracking accuracy remained higher in the finger tracking condition compared to the replay tracking condition even when blur disrupted tracking performance. The rate of decay of tracking accuracy beyond the critical blur level did not vary between finger and replay tracking conditions (finger vs replay main effect: $F_{1,4}=0.53, p=0.51$ ). The rate of decay of tracking accuracy was also similar for horizontal and vergence eye movements (horizontal vs vergence main effect: $F_{1,4}=1.23, p=$ $0.33)$. 
Monocular blur hinders vergence tracking, but not horizontal pursuit tracking

- The rate of decay of tracking accuracy differed across monocular and binocular blur conditions (monocular vs binocular blur main effect: $F_{1,4}=43.04, p=0.0028$ ), and more specifically as a function of whether tracking was left-right or in depth (interaction effect between monocular vs binocular blur and horizontal vs vergence: $F_{1,4}=9.87, p=0.035$ ). Figure $6 \mathrm{~b}$ shows that when observers executed horizontal pursuit eye movements (left), the rate of decay was steep (i.e., more negative) if blur was rendered to both eyes and shallow if blur was rendered to one eye only, and this difference was statistically significant; $t(4)=$ $-10.23, p=0.00052$, paired samples $t$ test, Cohen's $d=5.97$. When observers executed vergence eye movements instead, the rate of decay was similarly steep in both binocular and monocular blur conditions (as seen in the right panel of Fig. 6b); $t(4)=-0.99, p=0.38$, paired samples $t$ test, Cohen's $d=0.39$. All other ANOVA two- and three-way interactions were not statistically significant (all $p>0.19$ ).

Moderate amounts of blur impair tracking accuracy-The overall critical blur level at which tracking accuracy began to fall off was found to be $24 \operatorname{arcmin}(18-32 \operatorname{arcmin}, 95 \%$ confidence range). This critical blur value was independent of the type of eye movement executed (horizontal vs vergence main effect: $F_{1,4}=1.41, p=0.30$ ), of whether observers were tracking their own finger movements or a replay of previously executed finger movements (finger vs replay main effect: $F_{1,4}=0.81, p=0.42$ ) and of whether blur was rendered monocularly or binocularly (monocular vs binocular blur main effect: $F_{1,4}=1.031$, $p=0.37$; all two- and three-way interactions: $p>0.22$ ).

The rendered blur employed in this study was meant to simulate the effects of uncorrected refractive error. A very simple approximate relationship exists between blur in angular units and dioptric level of defocus (Smith 1982):

$$
\beta=p_{\mathrm{d}}\left(R-D_{\mathrm{t}}\right)
$$

meaning that defocus in angular units $\beta$ (radians) is equal to the pupil diameter $p_{\mathrm{d}}(\mathrm{m})$ multiplied by the difference between the refractive status of the eye $R$ (diopters) and the distance to the target $D_{\mathrm{t}}$ (diopters). Given that we have estimated the critical blur level at which tracking performance begins to deteriorate, we can employ Eq. (2) to estimate at what level of refractive error oculomotor tracking performance begins to break down.

Common refractive errors may impair oculomotor tracking-Figure $6 \mathrm{c}$ shows the estimated critical refraction as a function of pupil diameter for a target at $57 \mathrm{~cm}$ (dashed line; within arm's length) and for a target at optical infinity (continuous line; optical infinity in clinical practice is commonly defined as any distance farther than $6 \mathrm{~m}$ ). Pupil size is strongly dependent on light levels: pupils will constrict in high light and enlarge with low light levels. When visually tracking targets within arm's length, only relatively large refractive errors (> 5 diopters) will degrade oculomotor performance in outdoors, high light levels. In low light levels instead, 2.5 diopters of refractive error will already degrade oculomotor tracking of targets within arm's length. When looking at optical infinity in high, outdoor light levels, up to 3 diopters of refractive error may be necessary to hinder oculomotor performance. For 
low, indoor light levels instead, 1 diopter of refractive error may already be sufficient to degrade oculomotor tracking performance for targets father than $6 \mathrm{~m}$ away. Confronting these estimated values of critical refraction with the distribution of refractive errors found in the general population (Fig. 1) highlights that a significant portion of children and adults may experience deficits in oculomotor tracking when refraction is not appropriately corrected.

\section{Complementary findings from exploratory analyses}

The findings from exploratory analyses help further characterize the differences in tracking performance between the finger and replay conditions under different experimental conditions. Figure 7 shows single trial tracking data from a subset of experimental conditions for one representative observer. In the horizontal pursuit tracking conditions (Fig. 7a), individual trials lasted on average $3 \mathrm{~s}$ (2.5-3.6, 95\% confidence range), and mean finger/ target velocity was $5.4 \mathrm{deg} / \mathrm{s}(4.8-5.9,95 \%$ confidence range). Similarly, in the vergence pursuit tracking conditions (Fig. 7b), individual trials lasted on average $2.9 \mathrm{~s}(2.4-3.1,95 \%$ confidence range), and mean finger/target velocity was $5.8 \mathrm{deg} / \mathrm{s}(4.7-7.0,95 \% \mathrm{CI})$. It is obvious that the vergence data exhibit much more noise than the horizontal pursuit data, which is in line with the large difference in accuracy observed between the pursuit and vergence tracking conditions. Additionally, for both horizontal and vergence tracking, at all levels of monocular and binocular blur, the target (green curves) is more closely matched by the observer's gaze position in the finger tracking condition (black) compared to the replay tracking condition (red curves).

Sensorimotor coupling aids the localization of the target-Figure 8 shows the position error as a function of time from target movement onset for both horizontal pursuit (a) and vergence tracking (b), for the finger tracking condition in black and the replay tracking condition in red. The patterns of the error traces were qualitatively similar at all blur levels and in both the binocular and monocular blur conditions, with only the magnitude of the error varying in the different blur conditions. Thus, for simplicity we present the average error traces from all blur conditions. During horizontal pursuit tracking (Fig. 8a), observers anticipated the target movement onset (observe the positive deflection of the error traces at time zero), and this anticipatory deflection was more pronounced when observers were tracking their own finger (black curve) than when they were tracking a replay of their own previously executed finger movements (red curve). After movement onset, the tracking error remained markedly smaller in the finger tracking condition (black curve) than in the replay tracking conditions (red curve). During vergence pursuit tracking (Fig. 8b), observers did not visibly anticipate the target movement onset in either the finger or the replay tracking conditions. However, after target movement onset, the tracking error was smaller during the finger tracking condition (black curve) than in the replay tracking conditions (red curve).

\section{The eyes are nearly synchronous to the hand during horizontal pursuit} tracking-To more closely investigate tracking performance at target movement onset, we measured the latency of gaze tracking in each experimental condition. Figure 9a shows how during horizontal pursuit tracking (black data), the eyes moved in almost perfect synchrony with the target during the finger tracking condition, whereas there was an average latency of 
$\approx 70 \mathrm{~ms}$ in the replay tracking condition. ANOVA analysis confirmed that horizontal pursuit tracking exhibited a significantly shorter latency in the finger tracking condition compared to the replay tracking condition (Finger vs Replay main effect: $F_{1,4}=32.7466, p=0.0046$, Cohen's $d=2.45$ ). The latency of horizontal pursuit tracking did not vary as a function of blur level (blur level main effect: $F_{6,24}=0.70, p=0.65$ ), and was independent of whether blur was rendered monocularly or binocularly (monocular vs binocular blur main effect: $F_{1,4}$ $=2.10, p=0.22$ ). All ANOVA two- and three-way interactions were also not statistically significant (all $p>0.082$ ).

\section{Sensorimotor coupling facilitates the prediction of target movement onset} also during vergence tracking-During vergence pursuit, as shown in Fig. 9b, gaze tracking was always delayed with respect to the onset of the target motion. However, tracking latency was noticeably smaller during finger tracking (black data, on average $\approx 100$ $\mathrm{ms}$ ) and larger in replay tracking (red data, on average $\approx 140 \mathrm{~ms}$ ). ANOVA analysis further confirmed statistically significant difference in vergence pursuit latency between finger and replay tracking conditions (Finger vs Replay main effect: $F_{1,4}=9.48, p=0.034$, Cohen's $d$ $=1.33$ ). As with horizontal pursuit tracking, the latency of vergence pursuit tracking did not vary with blur level (blur level main effect: $F_{5,20}=0.51, p=0.77$ ) nor whether blur was rendered monocularly or binocularly (monocular vs binocular blur main effect: $F_{1,4}=0.021$, $p=0.89$ ). ANOVA analysis revealed no significant two- or three-way interaction (all $p>$ $0.49)$.

\section{Fewer catch-up saccades occur during tracking of self-generated target}

motion-To investigate errors arising from difficulties in perceiving the velocity of motion along the fronto-parallel or sagittal planes, we measured the rate of saccades occurring during each tracking trial. During horizontal pursuit tracking, we measured the rate of catchup saccades that subjects executed to bring the two foveae back onto the target. Figure 10a shows that saccade rate was significantly smaller in the finger tracking condition (black) compared to the replay tracking condition (red; Finger vs Replay main effect: $F_{1,4}=37.11, p$ $=0.0037$, Cohen's $d=1.01$ ). We found no significant main effect of monocular vs binocular blur conditions $\left(F_{1,4}=4.55, p=0.10\right)$. There was however significant main effect of blur level ( $\left.F_{6,24}=5.77, p=0.00078\right)$ and significant interactions effects between finger vs replay and monocular vs binocular conditions $\left(F_{1,4}=18.67, p=0.012\right)$ and between monocular vs binocular conditions and blur Level $\left(F_{6,24}=7.86, p=0.000093\right)$. These significant effects were all driven by the $200 \mathrm{arcmin}$, binocular blur condition, in which the average saccade rate fell significantly below the average saccade rate from all other experimental conditions $[t(68)=-4.05, p=0.00013$, unpaired samples $t$ test; compare brown circle and star symbols in Fig. 10a]. There was no significant interaction between finger vs replay conditions and blur Level $\left(F_{6,24}=1.74, p=0.15\right)$, nor a significant three-way interaction $\left(F_{6,24}=1.06, p=\right.$ $0.41)$.

\section{Eye-hand coupling also increases the stability of vergence tracking-As}

expected, saccade rate in the vergence pursuit condition (Fig. 10b) was lower than in the horizontal pursuit condition, as observers were required to track a stimulus which only moved in depth and not left-right. Nevertheless, Fig. 10b shows how saccade rate varied as a 
function of blur level (blur level main effect: $F_{5,20}=17.16, p=0.0000013$ ), and specifically, saccade rate increased significantly from baseline when observers were presented with 50 $\operatorname{arcmin}(0$ vs 50 arcmin blur: $t(4)=-8.33, p=0.0057$, Bonferroni-corrected paired samples $t$ test, Cohen's $d=1.51$ ) and 100 arcmin of blur ( 0 vs 100 arcmin blur: $t(4)=-6.00, p=$ 0.019 , Cohen's $d=2.76$ ). Additionally, we found that saccade rate was statistically smaller during finger tracking than in the replay tracking (compare the black and red filled circles in Fig. 10b) (finger vs replay main effect: $F_{1,4}=12.21, p=0.025$, Cohen's $d=1.2832$ ). However, no significant difference was found between monocular and binocular blur conditions (monocular vs binocular blur main effect: $F_{1,4}=2.11, p=0.22$ ) and no significant two- or three-way interaction effects were found (all $p>0.21$ ).

\section{Discussion}

We investigated the link between the oculomotor and hand motor control systems under binocularly asymmetric blur conditions, for both horizontal pursuit eye movements and vergence pursuit eye movements in depth. We replicated the classic results that smooth pursuit eye movements to self-generated horizontal motion are more accurate than to externally generated motion (Steinbach and Held 1968). We extend these classic studies to show that the link between the eye and hand motor systems also exists for eye and hand movements in depth. Additionally, we show that moderate levels of simulated blur could disrupt oculomotor tracking performance, yet the link between the eye and hand motor system persists at all blur levels. Lastly, monocular and binocular blur have different effects on eye movements in the fronto-parallel (horizontal pursuit eye movements) and sagittal (vergence pursuit eye movements in depth) planes: binocular blur similarly disrupts both horizontal pursuit and vergence pursuit eye movements. On the other hand, monocular blur strongly affects vergence eye movements while only mildly affecting horizontal pursuit eye movements.

To elucidate the potential mechanisms underlying our primary results, we further characterized the patterns of tracking errors in our data using exploratory analyses. These analyses reveal that the linkage between the oculomotor and hand motor control systems helps predict the onset of target movement and localize the target position during movement tracking. In other words, when observers tracked their own finger, tracking became more stable with anticipated eye movements (as shown by a reduction in both tracking latency and saccade rate), compared to when observers tracked an externally moving target. These patterns held true for both horizontal pursuit eye movements and vergence pursuit eye movements in depth.

\section{Mechanisms of eye-hand coordination}

\section{Shared motor planning between the eyes and hands-It is possible that} proprioceptive information about hand location may play a role in guiding eye movements (Mather and Lackner 1980, 1981). However, proprioception does not fully account for increased accuracy at tracking self motion, since in patients devoid of proprioception pursuit movements are still able to anticipate the start of target motion when the motion is selfgenerated (Vercher et al. 1996). Thus, it is more likely that the eye and hand systems share 
motor planning information that facilitates the initiation of tracking eye movements, and then hand and arm proprioception helps maintain oculomotor tracking accuracy throughout the execution of the eye movements (Vercher and Gauthier 1992; Vercher et al. 1995, 1996; Lazzari et al. 1997). For example, a recent study (Chen et al. 2016) has shown that the lateralized readiness potential, which signals hand motor preparation, is associated with anticipatory smooth pursuit eye movements to self-generated finger motion. These findings provide convincing evidence that the hand motor system shares motor planning information with the oculomotor system: shared motor planning information could take the shape of a common command signal sent to both motor systems (Bock 1987). Alternatively, a coordination control system has been proposed to explain enhanced accuracy at tracking self motion, in which motor efference copy is employed to synchronize the oculomotor and hand motor systems (Gauthier et al. 1988). This coordination control model is supported by the near-perfect synchrony of eye and hand movement onset in smooth pursuit studies, because if oculomotor and hand motor system received a common command signal, the differences (both biomechanical and neural) between these motor systems would lead to asynchronies in the hand and eye movement onsets (Scarchilli and Vercher 1999). In our study, horizontal pursuit eye movements were initiated almost exactly at the same time as the target motion in the finger tracking trials, which supports the notion that oculomotor tracking is synchronized with the target through hand motor efference copy. However, this did not hold true for vergence tracking eye movements, where the eyes always lagged behind the target motion onset even though tracking latency was reduced during finger tracking compared to replay tracking. The different latencies found for horizontal pursuit and vergenge tracking eye movements are consistent with known differences in the spatio-temporal characteristics of these different eye movements (Semmlow et al. 1998; Lisberger 2015; Yang et al. 2002) and could reflect differences in the neural substrates underlying sensorimotor coupling for coordinated hand-eye movements in depth or along the frontal plane. The cerebellum, which is directly involved in the control of both pursuit (Westheimer and Blair 1974; Thier and Ilg 2005) and vergence (Gamlin et al. 1996; Gamlin 2002) eye movements, likely plays an important role in the synchronization of the oculomotor and arm motor systems (Miall et al. 2000, 2001). If the same synchronization signals from the cerebellum were to converge onto separate vergence and pursuit-specific neural loci (Gamlin and Yoon 2000; Ward et al.

2015), these signals might differentially impact oculomotor tracking performance in depth and along the frontal plane.

Afferent signals from the hand reduce oculomotor tracking errors-The role of arm proprioceptive signal in maintaining oculomotor tracking accuracy is demonstrated by studies showing that, when visually tracking externally generated motion, manual tracking enhanced smooth pursuit eye movements (at least when the object motion was predictable) (Koken and Erkelens 1992; Niehorster et al. 2015). Koken and Erkelens (1993) have however shown that when observers executed vergence eye movements to track a stimulus moving predictably in depth under external control, concurrent hand tracking of the stimulus did not aid oculomotor tracking performance. These results suggest that the oculomotor system may employ limb proprioceptive (afferent) signals to plan eye movements in the fronto-parallel plane, but might be unable to do so for eye movements in depth. In the current study, we find that tracking accuracy is higher for both horizontal pursuit and 
vergence eye movements when observers track self-generated hand motion. More importantly, this finding is not only due to better predictions of movement onset, but also due to a better estimation of the target motion during tracking, as demonstrated by smaller tracking errors (Fig. 8) and increased tracking stability (i.e., smaller saccade rate, Fig. 10) in the finger tracking conditions for both pursuit and vergence tracking. Thus, our findings challenge previous findings (Koken and Erkelens 1993) by suggesting that both efferent and afferent signals from the hand motor system may play a critical role in maintaining the accuracy of both horizontal pursuit and vergence tracking eye movements in depth.

The strength of sensorimotor coupling may remain invariant-If the oculomotor system were able to modulate the contributions of the visual input and the hand motor efferent/afferent signals when planning horizontal smooth pursuit and vergence tracking eye movements, we might expect the hand motor signals to be weighed more strongly in conditions of greater visual uncertainty. However, the rate of decay of tracking accuracy remained the same in the finger and replay tracking conditions. Taken together, the contribution of the hand motor signals to oculomotor planning appears to be invariant over the short times covered in the present study. We, however, cannot rule out the possibility that the contribution of motor efferent/afferent signals may change over longer periods of adaptation to visual impairment.

Only binocularly symmetric blur impairs fronto-parallel tracking-It should be noted that the rendered blur employed in our study reduced both the contrast and the spatial frequency content of the visual target. When blurred stimuli were presented to one eye only, it is possible that the interocular differences in contrast and spatial frequency content could trigger interocular suppression (Fahle 1982; Kwon et al. 2015). Hence, we can speculate that with increasing blur level, the impoverished visual input to the blurred eye was likely to be suppressed. Previous studies have shown that reduced contrast impairs pursuit eye movements, whereas changes in spatial frequency have no consistent effect on frontoparallel tracking performance (Spering et al. 2005). Our data indeed show a marked reduction in accuracy for horizontal pursuit tracking at increasing binocular blur levels, consistent with what we expected from the reduced contrast of a target. On the other hand, horizontal pursuit tracking was not strongly affected by monocular blur, as oculomotor performance likely relied on the eye viewing the non-blurred target. In the case of horizontal pursuit tracking, suppression of the blurred eye might actually aid tracking performance by reducing the noise in the system. However, the strength of interocular suppression is known to fluctuate on short timescales (de Belsunce and Sireteanu 1991). Hence, our tracking data in the monocular blur, horizontal tracking condition (Fig. 5b), which exhibited small fluctuations in accuracy (that were not well captured by the hinged-line model), might have been influenced by fluctuations in the strength of interocular suppression.

\section{Both binocularly symmetric and asymmetric blur impair vergence tracking in}

depth-In vergence eye movements, the images projected onto the foveae of the two eyes need to be aligned and thus high spatial frequency content in the two eyes is required to be matched (Schor et al. 1984; Lam et al. 1996; Judge 1996; Maiello et al. 2014). The vergence system is also tightly linked to the accommodation system, which attempts to minimize 
visual blur at the fovea of both eyes (Fincham and Walton 1957; Judge 1996). In particular, some pre-motor vergence neurons may attempt to combine disparity and blur information to drive vergence, and under conditions of either monocular or binocular blur these neurons will receive a mismatched drive from 'blur' and disparity (Judge and Cumming 1986). Hence, in our study vergence tracking was always strongly impaired by high levels of blur, because in both monocular and binocular blur conditions the matching low spatial frequency information from the two eyes was insufficient to drive accurate binocular alignment. Our statistical analyses show no significant differences in vergence tracking performance between monocular and binocular blur conditions. Nevertheless, Fig. 5 shows an interesting pattern of results, in which a decrease in the performance of vergence eye movements appeared to be more pronounced at high levels of monocular blur as compared to at high levels of binocular blur. This pattern of data, which is the opposite to that observed for horizontal pursuit, is also consistent with suppression of the eye experiencing impoverished visual input. At high levels of monocular blur, interocular suppression could mask the remaining matching binocular input altogether, making binocular alignment essentially impossible.

\section{Limitations and future directions}

We acknowledge some methodological limitations of our work which may be relevant to future research into sensorimotor coupling. In the current study, motion of the index finger determined visual target motion, yet the target was in a different position with respect to the finger. Additionally, the low-cost finger tracking device employed in the current study introduces a lag between the time when the finger actually moves and when the target motion moves on-screen. As such, these conditions may not faithfully represent the normal coordination between oculomotor and hand movement control systems. Nevertheless, our data and results are highly consistent with the previous findings reported by other groups (Steinbach and Held 1968; Gauthier et al. 1988; Vercher and Gauthier 1992; Koken and Erkelens 1992; Vercher et al. 1995, 1996; Lazzari et al. 1997; Scarchilli and Vercher 1999; Niehorster et al. 2015; Chen et al. 2016). It should be also noted that our tracking latency data did not increase with increasing visual uncertainty (e.g., blur level in either horizontal or vergence pursuit tracking). However, this may be largely due to the predictability of the target movement, as previously suggested by Chen et al. (2016). Overall, our data suggest that eye-hand coordination need not be spatially and temporally aligned, and future work is needed to characterize the spatio-temporal limits of sensorimotor coupling.

Another technological limitation lies in the fact that shutter glasses were used in the current study to render stereoscopic depth and to dichoptically vary the blur simulated in the two eyes. This could have impacted eye tracking data quality, since the eye tracker viewed the observer's eyes through the shutter glasses. However, this method has been successfully used in our published gaze-contingent display (Maiello et al. 2014) and saccadic adaptation (Maiello et al. 2016) studies, both of which required highly accurate binocular eye movement recordings. In addition, simulating depth through shutter glasses can potentially decouple vergence and accommodation (Rushton and Riddell 1999; Hoffman et al. 2008; Wann et al. 1995), which might induce additional noise in the vergence-accommodation system. We, however, found that sensorimotor coupling survived this potential cue conflict. 
Nevertheless, the role of conflicting disparity and focus cues in virtual reality technology with respect to eye-hand coordination requires further investigation in relation to refractive error development (Maiello et al. 2018), as we discuss further below.

It is also important to note that in the current study visual impairments were simulated by Gaussian blurring the visual input. It is possible that oculomotor performance may be affected by different kinds of blur (such as sinc blur which contains phase reversals typical of the modulation transfer function of an optical system with a circular aperture such as the human pupil, Murray and Bex 2010), and that observers may learn to adapt to the specific type of defocus blur arising from their own optics (Artal et al. 2004). Future investigations should thus consider to examine hand-eye coordination and oculomotor control in the general population as well as monocular visual acuity for static optotypes. Furthermore, as our blur manipulation affects both the contrast and the spatial frequency content of the visual targets, in a future study it would be helpful to directly tease apart the contributions of contrast and spatial frequency to oculomotor control and eye-hand coordination in 3D.

A final note concerns the fact that all observers in our study were right eye dominant, and monocular blur was always presented to their non-dominant left eye. Interocular differences in contrast sensitivity and acuity due to eye dominance are measurable in normally sighted observers (Kwon et al. 2015). These differences are indeed known to affect stereoacuity (Lam et al. 1996), and to be associated with phoria, i.e., the amount of ocular deviation occurring when fixating a target with one occluded eye (Ono and Weber 1981). As binocular rivalry suppression is known to be skewed in favor of the dominant eye (Handa et al. 2004), a future study should consider the potential role of eye dominance.

\section{Clinical significance of our findings}

\section{Uncorrected refractive errors can significantly impair oculomotor} performance-The critical blur level at which tracking accuracy began to decay was independent of all experimental manipulations in the present study. Furthermore, our additional analysis demonstrates that refractive errors between 1 and 5 diopters may be sufficient enough to produce this level of critical blur, depending on the ambient light level and viewing distance. Consider this range of critical refraction with respect to the distribution of refractive errors found in the general population (Fig. 1). A significant portion of the population has refractive errors that, in our study, correspond to a decrease in oculomotor tracking performance. Furthermore, individuals with amblyopia or those with binocularly asymmetric visual impairment (such as cataracts), not only have an average acuity loss beyond this level of critical blur, but even the average difference in refraction between the amblyopic and fellow eye (1.3 diopters) might be sufficient to impair oculomotor performance. Our findings are thus in line with multiple studies showing that eye-hand coordination skills are significantly impaired in children with amblyopia as compared to normal cohorts (Grant et al. 2014; Suttle et al. 2011; Sloper et al. 2011).

\section{Binocular eye movements and visual processing may synergistically degrade} in amblyopia-More importantly, our findings may help us better understand the aetiology of amblyopia. It has been thought that this visual dysfunction develops when binocular 
vision becomes decorrelated owing to either ocular misalignment or interocular difference in refractive error, in which the brain learns to suppress visual signals from the weak eye to avoid diplopia (double vision) (Wright et al. 2006). More specifically, uniocular blur in early life has been shown to induce amblyopia in macaque monkeys by impairing the spatial resolution and contrast sensitivity of the affected eye (Kiorpes et al. 1987; Hendrickson et al. 1987). Importantly, uniocular blur also reduces the degree of binocular interaction throughout the visual pathways, a necessary component of binocular oculomotor control (Movshon et al. 1987). However, the development of oculomotor and perceptual deficits in amblyopia might be interdependent. If the visual input from one eye is not correlated with the other eye, our findings show that oculomotor control might begin to degrade. Vergence eye movements are likely to become less accurate (Schor et al. 1984; Judge 1996; Maiello et al. 2014, 2016), but also the mechanisms that calibrate the accuracy of binocular saccades might begin to fail (Albano and Marrero 1995; Schultz and Busettini 2013; Maiello et al. 2016). If this occurs during early life, when vision and oculomotor control are in development (Atkinson et al. 1977; Banks and Salapatek 1978; Roucoux et al. 1983; Hainline et al. 1984; Hainline and Riddell 1995), oculomotor and perceptual deficits might exacerbate each other. Binocularly imbalanced visual input may lead to poor binocular oculomotor control. Impaired oculomotor control in terms of imprecise binocular alignment, inaccurate binocular saccades, and incorrect eye posture (ocular deviation) will further decorrelate the visual input to the two eyes. This increased decorrelation between the visual input to the two eyes may lead to increased suppression of the weak eye. Increased suppression may in turn exacerbate oculomotor deficits, and so on. Thus, not only may the perceptual deficits occurring in amblyopia lead to oculomotor deficits: perceptual and oculomotor deficits together might form a feedback loop of visuomotor impairment.

Eye-hand coordination for visual rehabilitation-This work also has potential implications for visual rehabilitation strategies in which binocular vision and eye movements are impaired, such as amblyopia, strabismus and convergence insufficiency. Classical patching therapy might be counterproductive for the development of coordinated vergence eye movements because it forces the two eyes to work independently and does not favor the correct development of binocular oculomotor control. Dichoptic therapies that attempt to balance the input to the two eyes and favor conjugate eye movements might be better suited to ensure binocular cooperation (Hess et al. 2010; To et al. 2011; Knox et al. 2012; Li et al. 2013; Tailor et al. 2015, 2016; Maiello et al. 2016; Bossi et al. 2017). Here, we have shown that the link between the oculomotor and hand motor system is unaffected by simulated visual impairment. Thus, eye-hand coordination tasks (Vedamurthy et al. 2016) might be able to provide a boost to visual rehabilitation strategies by enhancing oculomotor control both along the fronto-parallel plane and particularly in depth. This tantalizing idea is further supported by the notion that visual processing in general, not solely oculomotor performance, is enhanced near the hand (which suggests some cross-modality overlap of spatial representations). For example, several studies have demonstrated that visual stimuli placed near the hands are more readily detected (Craighero et al. 1999; Reed et al. 2006; Jackson et al. 2010; Perry et al. 2016), importantly even in visually impaired neurological patients (Di Pellegrino and Frassinetti 2000; Schendel and Robertson 2004; Brown et al. 2008). Perry et al. (2015) have shown that the orientation selectivity of V2 neurons is 
enhanced for stimuli near the hand via the sharpening of orientation tuning. The effects of hand proximity have not yet been thoroughly investigated for other perceptual dimensions, such as visual processing of motion and depth, which are highly relevant in binocular visual deficits. Future studies should therefore focus on determining whether hand proximity may favor binocular cooperation. If this were the case, then eye-hand coordination tasks could be employed to synergistically enhance both binocular oculomotor performance and binocular visual perception, thus significantly augmenting current visual rehabilitation strategies.

\section{Acknowledgements}

The authors thank Dr. Jing Chen for help with setting up the pursuit onset detection algorithm originally developed by Dr. Alexander Schtz, as well as the two anonymous reviewers. This research was supported by the National Institutes of Health Grant R01EY021553. Data andanalysis scripts are available from the Zenodo database (https:// doi.org/10.5281/zenodo.1100971).

\section{References}

Akao T, Kurkin SA, Fukushima J, Fukushima K (2005) Visual and vergence eye movement-related responses of pursuit neurons in the caudal frontal eye fields to motion-in-depth stimuli. Exp Brain Res 164(1):92-108 [PubMed: 15924235]

Albano JE, Marrero JA (1995) Binocular interactions in rapid saccadic adaptation. Vis Res 35(23): 3439-3450 [PubMed: 8560810]

Artal P, Chen L, Fernández EJ, Singer B, Manzanera S, Williams DR (2004) Neural compensation for the eye's optical aberrations. J Vis 4(4):4-4

Atkinson J, Braddick O, Moar K (1977) Development of contrast sensitivity over the first 3 months of life in the human infant. Vis Res 17(9):1037-1044 [PubMed: 595412]

Banks M, Salapatek P (1978) Acuity and contrast sensitivity in 1-, 2-, and 3-month-old human infants. Investig Ophthalmol Vis Sci 17(4):361-365 [PubMed: 640783]

Bex PJ, Makous W (2002) Spatial frequency, phase, and the contrast of natural images. J Opt Soc Am A 19(6):1096-1106

Bock O (1987) Coordination of arm and eye movements in tracking of sinusoidally moving targets. Behav Brain Res 24(2):93-100 [PubMed: 3593529]

Bossi M, Tailor VK, Anderson EJ, Bex PJ, Greenwood JA, Dahlmann-Noor A, Dakin SC (2017) Binocular therapy for childhood amblyopia improves vision without breaking interocular suppression. Investig Ophthalmol Vis Sci 58(7):3031 [PubMed: 28614556]

Brainard DH (1997) The psychophysics toolbox. Spat Vis 10:433-436 [PubMed: 9176952]

Brown LE, Kroliczak G, Demonet JF, Goodale MA (2008) A hand in blindsight: hand placement near target improves size perception in the blind visual field. Neuropsychologia 46(3):786-802 [PubMed: 18206962]

Brown MA, Stuerzlinger W, Filho EJM (2014) The performance of un-instrumented in-air pointing. In: Proceedings of graphics interface 2014, GI ‘14. Canadian Information Processing Society, Toronto, Ont., Canada, pp 59-66

Chen J, Valsecchi M, Gegenfurtner KR (2016) LRP predicts smooth pursuit eye movement onset during the ocular tracking of self-generated movements. J Neurophysiol

Cornelissen FW, Peters EM, Palmer J (2002) The eyelink toolbox: eye tracking with matlab and the psychophysics toolbox. Behav Res Methods Instrum Comput 34(4):613-617 [PubMed: 12564564]

Coubard OA (2013) Saccade and vergence eye movements: a review of motor and premotor commands. Eur J Neurosci 38(10):3384-3397 [PubMed: 24103028]

Craighero L, Fadiga L, Rizzolatti G, Umiltà C (1999) Action for perception: a motor-visual attentional effect. J Exp Psychol Hum Percept Perform 25(6):1673 [PubMed: 10641315]

de Belsunce S, Sireteanu R (1991) The time course of interocular suppression in normal and amblyopic subjects. Investig Ophthalmol Vis Sci 32(9):2645 [PubMed: 1869416] 
Di Pellegrino G, Frassinetti F (2000) Direct evidence from parietal extinction of enhancement of visual attention near a visible hand. Curr Biol 10(22):1475-1477 [PubMed: 11102814]

Ellis SR, Breant F, Manges B, Jacoby R, Adelstein BD (1997) Factors influencing operator interaction with virtual objects viewed via head-mounted see-through displays: viewing conditions and rendering latency. In: Proceedings of IEEE 1997 annual international symposium on virtual reality, pp 138-145

Fahle M (1982) Binocular rivalry: suppression depends on orientation and spatial frequency. Vis Res 22(7):787-800 [PubMed: 7123863]

Fan D, Cheung E, Lai R, Kwok A, Lam D (2004) Myopia progression among preschool chinese children in hong kong. Ann Acad Med Singapore 33(1):39-43 [PubMed: 15008560]

Fielder AR, Moseley MJ (1996) Does stereopsis matter in humans? Eye 10(2):233-238 [PubMed: 8776453]

Fincham E, Walton J (1957) The reciprocal actions of accommodation and convergence. J Physiol 137(3):488 [PubMed: 13463783]

Fisher RA (1915) Frequency distribution of the values of the correlation coefficient in samples from an indefinitely large population. Biometrika 10(4):507-521

Fukushima K, Yamanobe T, Shinmei Y, Fukushima J, Kurkin S, Peterson BW (2002) Coding of smooth eye movements in three-dimensional space by frontal cortex. Nature 419(6903):157-162 [PubMed: 12226663]

Gamlin PD, Yoon K (2000) An area for vergence eye movement in primate frontal cortex. Nature 407(6807):1003-1007 [PubMed: 11069179]

Gamlin PDR (2002) Neural mechanisms for the control of vergence eye movements. Ann N Y Acad Sci 956(1):264-272 [PubMed: 11960810]

Gamlin PDR, Yoon K, Zhang H (1996) The role of cerebro-pontocerebellar pathways in the control of vergence eye movements. Eye 10(2):167-171 [PubMed: 8776444]

Gauthier G, Vercher JL, Ivaldi FM, Marchetti E (1988) Oculo-manual tracking of visual targets: control learning, coordination control and coordination model. Exp Brain Res 73(1):127-137 [PubMed: 3208852]

Gonzalez DA, Niechwiej-Szwedo E (2016) The effects of monocular viewing on hand-eye coordination during sequential grasping and placing movements. Vis Res 128:30-38 [PubMed: 27664351]

Grant S, Suttle C, Melmoth DR, Conway ML, Sloper JJ (2014) Age- and stereovision-dependent eyehand coordination deficits in children with amblyopia and abnormal binocularity. Investig Ophthalmol Vis Sci 55(9):5687 [PubMed: 25097239]

Guna J, Jakus G, Pogačnik M, Tomažič S, Sodnik J (2014) An analysis of the precision and reliability of the leap motion sensor and its suitability for static and dynamic tracking. Sensors 14(2):37023720 [PubMed: 24566635]

Hainline L, Riddell PM (1995) Binocular alignment and vergence in early infancy. Vis Res 35(23): 3229-3236 [PubMed: 8560794]

Hainline L, Turkel J, Abramov I, Lemerise E, Harris CM (1984) Characteristics of saccades in human infants. Vis Res 24(12):1771-1780 [PubMed: 6534000]

Handa T, Mukuno K, Uozato H, Niida T, Shoji N, Shimizu K (2004) Effects of dominant and nondominant eyes in binocular rivalry. Optom Vis Sci 81(5):377-383 [PubMed: 15181364]

Hendrickson AE, Movshon J, Eggers HM, Gizzi MS, Boothe R, Kiorpes L (1987) Effects of early unilateral blur on the macaque's visual system. II. Anatomical observations. J Neurosci 7(5):13271339 [PubMed: 3033169]

Hess R, Mansouri B, Thompson B (2010) A new binocular approach to the treatment of amblyopia in adults well beyond the critical period of visual development. Restor Neurol Neurosci 28(6):793802 [PubMed: 21209494]

Hoffman DM, Girshick AR, Akeley K, Banks MS (2008) Vergence-accommodation conflicts hinder visual performance and cause visual fatigue. J Vis 8(3):33

Hyams S, Pokotilo E, Shkurko G (1977) Prevalence of refractive errors in adults over 40: a survey of 8102 eyes. Br J Ophthalmol 61(6):428-432 [PubMed: 871471]

Exp Brain Res. Author manuscript; available in PMC 2019 August 14. 
Jackson CP, Miall RC, Balslev D (2010) Spatially valid proprioceptive cues improve the detection of a visual stimulus. Exp Brain Res 205(1):31-40 [PubMed: 20567807]

Judge SJ (1996) How is binocularity maintained during convergence and divergence? Eye 10(2):172176 [PubMed: 8776445]

Judge SJ, Cumming BG (1986) Neurons in the monkey midbrain with activity related to vergence eye movement and accommodation. J Neurophysiol 55(5):915-930 [PubMed: 3711972]

Kamiya S (1984) Analysis of school myopia using the nidek auto-refractometer ar 3000. Part 2. Observation of frequency distribution of pupil refraction. Folia Ophthalmol Jpn 35:1755-1769

Kiorpes L, Boothe R, Hendrickson A, Movshon JA, Eggers HM, Gizzi M (1987) Effects of early unilateral blur on the Macaque's visual system. I. Behavioral observations. J Neurosci 7(5):13181326 [PubMed: 3572483]

Knox PJ, Simmers AJ, Gray LS, Cleary M (2012) An exploratory study: prolonged periods of binocular stimulation can provide an effective treatment for childhood amblyopia. Investig Ophthalmol Vis Sci 53(2):817-824 [PubMed: 22169103]

Koken PW, Erkelens CJ (1992) Influences of hand movements on eye movements in tracking tasks in man. Exp Brain Res 88(3):657-664 [PubMed: 1587324]

Koken PW, Erkelens CJ (1993) Simultaneous hand tracking does not affect human vergence pursuit. Exp Brain Res 96(3):494-500 [PubMed: 8299750]

Kotecha A, O'Leary N, Melmoth D, Grant S, Crabb DP (2009) The functional consequences of glaucoma for eyehand coordination. Investig Ophthalmol Vis Sci 50(1):203 [PubMed: 18806294]

Kretzmer ER (1952) Statistics of television signals. Bell Syst Tech J 31(4):751-763

Kwon M, Wiecek E, Dakin SC, Bex PJ (2015) Spatial-frequency dependent binocular imbalance in amblyopia. Sci Rep 5:17181 [PubMed: 26603125]

Lam AKC, Chau ASY, Lam WY, Leung GY, Man BSH (1996) Effect of naturally occurring visual acuity differences between two eyes in stereoacuity. Ophthal Physiol Opt 16(3):189-195

Lazzari S, Vercher JL, Buizza A (1997) Manuo-ocular coordination in target tracking. I. A model simulating human performance. Biol Cybern 77(4):257-266 [PubMed: 9394443]

Li J, Thompson B, Deng D, Chan LY, Yu M, Hess RF (2013) Dichoptic training enables the adult amblyopic brain to learn. Curr Biol 23(8):R308-R309 [PubMed: 23618662]

Lisberger SG (2015) Visual guidance of smooth pursuit eye movements. Annu Rev Vis Sci 1:447-468 [PubMed: 28532366]

Maiello G, Chessa M, Solari F, Bex PJ (2014) Simulated disparity and peripheral blur interact during binocular fusion. J Vis 14(8):13

Maiello G, Gibaldi APSS, Bex PJ (2016) Vergence eye movements to unbalanced dichoptic visual stimuli. In: 39th European conference on visual perception (ECVP) 2016 Barcelona, Perception, vol 45, pp 79-79

Maiello G, Harrison WJ, Bex PJ (2016) Monocular and binocular contributions to oculomotor plasticity. Sci Rep 6:31861 [PubMed: 27535336]

Maiello G, Kerber KL, Thorn F, Bex PJ, Vera-Diaz FA (2018) Vergence driven accommodation with simulated disparity in myopia and emmetropia. Exp Eye Res 166:96-105 [PubMed: 29051012]

Maiello G, Kwon M, Bex PJ (2016) 3 dimensional binocular eye and hand coordination in normal vision and with simulated visual impairments. J Vis 16(12):22-22

Mather JA, Lackner JR (1980) Visual tracking of active and passive movements of the hand. Q J Exp Psychol 32(2):307-315 [PubMed: 7433622]

Mather JA, Lackner JR (1981) The influence of efferent, proprioceptive, and timing factors on the accuracy of eye-hand tracking. Exp Brain Res 43(3-4):406-412 [PubMed: 7262233]

Miall RC, Imamizu H, Miyauchi S (2000) Activation of the cerebellum in co-ordinated eye and hand tracking movements: an FMRI study. Exp Brain Res 135(1):22-33 [PubMed: 11104124]

Miall RC, Reckess GZ, Imamizu H (2001) The cerebellum coordinates eye and hand tracking movements. Nat Neurosci 4(6):638-644 [PubMed: 11369946]

Movshon JA, Eggers HM, Gizzi MS, Hendrickson AE, Kiorpes L, Boothe R (1987) Effects of early unilateral blur on the Macaque's visual system. III. Physiological observations. J Neurosci 7(5): 1340-1351 [PubMed: 3572484] 
Murray S, Bex PJ (2010) Perceived blur in naturally contoured images depends on phase. Front Psychol 1:185 [PubMed: 21833246]

Niehorster DC, Siu WWF, Li L (2015) Manual tracking enhances smooth pursuit eye movements. J Vis 15(15): 11

O'Connor AR, Birch EE, Anderson S, Draper H (2010) The functional significance of stereopsis. Investig Ophthalmol Vis Sci 51(4):2019-2023 [PubMed: 19933184]

Ono H, Weber EU (1981) Nonveridical visual direction produced by monocular viewing. J Exp Psychol Hum Percept Perform 7(5):937 [PubMed: 6457113]

Pelli DG (1997) The videotoolbox software for visual psychophysics: transforming numbers into movies. Spat Vis 10:437-442 [PubMed: 9176953]

Perry CJ, Amarasooriya P, Fallah M (2016) An eye in the palm of your hand: alterations in visual processing near the hand, a minireview. Front Comput Neurosci:10

Perry CJ, Sergio LE, Crawford JD, Fallah M (2015) Hand placement near the visual stimulus improves orientation selectivity in v2 neurons. J Neurophysiol 113(7):2859-2870 [PubMed: 25717165]

Reed CL, Grubb JD, Steele C (2006) Hands up: attentional prioritization of space near the hand. J Exp Psychol Hum Percept Perform 32(1):166 [PubMed: 16478334]

Resnikoff S, Pascolini D, Mariotti SP, Pokharel GP (2008) Global magnitude of visual impairment caused by uncorrected refractive errors in 2004. Bull World Health Organ 86(1):63-70 [PubMed: 18235892]

Roucoux A, Culee C, Roucoux M (1983) Development of fixation and pursuit eye movements in human infants. Behav Brain Res 10(1):133-139 [PubMed: 6639721]

Rushton SK, Riddell PM (1999) Developing visual systems and exposure to virtual reality and stereo displays: some concerns and speculations about the demands on accommodation and vergence. Appl Ergon 30(1):69-78 [PubMed: 10098818]

Scarchilli K, Vercher JL (1999) The oculomanual coordination control center takes into account the mechanical properties of the arm. Exp Brain Res 124(1):42-52 [PubMed: 9928788]

Schendel K, Robertson LC (2004) Reaching out to see: arm position can attenuate human visual loss. J Cogn Neurosci 16(6):935-943 [PubMed: 15298781]

Schor C, Wood I, Ogawa J (1984) Binocular sensory fusion is limited by spatial resolution. Vis Res 24(7):661-665 [PubMed: 6464360]

Schultz KP, Busettini C (2013) Short-term saccadic adaptation in the macaque monkey: a binocular mechanism. J Neurophysiol 109(2):518-545 [PubMed: 23076111]

Schütz AC, Delipetkos E, Braun DI, Kerzel D, Gegenfurtner KR (2007) Temporal contrast sensitivity during smooth pursuit eye movements. J Vis 7(13):3

Semmlow JL, Yuan W, Alvarez TL (1998) Evidence for separate control of slow version and vergence eye movements: support for hering's law. Vis Res 38(8):1145-1152 [PubMed: 9666973]

Sloper JJ, Suttle CM, Finlay AL, Melmoth DR, Grant S (2011) Impaired development of eye-hand coordination in children with strabismus and amblyopia. J Am Assoc Pediatr Ophthalmol Strabismus 15(1):e8

Smith G (1982) Angular diameter of defocus blur discs. Optom Vis Sci 59(11):885-889

Sorsby A, Sheridan M, Leary GA, Benjamin B (1960) Vision, visual acuity, and ocular refraction of young men. Br Med J 1(5183):1394 [PubMed: 13832932]

Spering M, Kerzel D, Braun DI, Hawken MJ, Gegenfurtner KR (2005) Effects of contrast on smooth pursuit eye movements. J Vis 5(5):6

Steinbach MJ, Held R (1968) Eye tracking of observer-generated target movements. Science 161(3837):187-188 [PubMed: 5657071]

Stewart C, Moseley M, Fielder A, Stephens D (2004) Refractive adaptation in amblyopia: quantification of effect and implications for practice. Br J Ophthalmol 88(12):1552-1556 [PubMed: 15548811]

Suttle CM, Melmoth DR, Finlay AL, Sloper JJ, Grant S (2011) Eye-hand coordination skills in children with and without amblyopia. Investig Ophthalmol Vis Sci 52(3):1851 [PubMed: 21212188]

Exp Brain Res. Author manuscript; available in PMC 2019 August 14. 
Tailor V, Bossi M, Bunce C, Greenwood JA, Dahlmann-Noor A (2015) Binocular versus standard occlusion or blurring treatment for unilateral amblyopia in children aged three to eight years. Cochrane Database Syst Rev 10.1002/14651858.CD011347.pub2

Tailor V, Bossi M, Greenwood JA, Dahlmann-Noor A (2016) Childhood amblyopia: current management and new trends. Br Med Bull 119(1):75-86. 10.1093/bmb/ldw030 [PubMed: 27543498]

Teather RJ, Pavlovych A, Stuerzlinger W, MacKenzie IS (2009) Effects of tracking technology, latency, and spatial jitter on object movement. In: 2009 IEEE symposium on 3D user interfaces, pp $43-50$

Thier P, Ilg UJ (2005) The neural basis of smooth-pursuit eye movements. Curr Opin Neurobiol 15(6): 645-652 [PubMed: 16271460]

To L, Thompson B, Blum JR, Maehara G, Hess RF, Cooperstock JR (2011) A game platform for treatment of amblyopia. IEEE Trans Neural Syst Rehabil Eng 19(3):280-289 [PubMed: 21335317]

Vedamurthy I, Knill DC, Huang SJ, Yung A, Ding J, Kwon OS, Bavelier D, Levi DM (2016) Recovering stereo vision by squashing virtual bugs in a virtual reality environment. Philos Trans $\mathrm{R}$ Soc B 371(1697):20150,264

Vercher JL, Gauthier G (1992) Oculo-manual coordination control: ocular and manual tracking of visual targets with delayed visual feedback of the hand motion. Exp Brain Res 90(3):599-609 [PubMed: 1426116]

Vercher JL, Gauthier GM, Guedon O, Blouin J, Cole J, Lamarre Y (1996) Self-moved target eye tracking in control and deafferented subjects: roles of arm motor command and proprioception in arm-eye coordination. J Neurophysiol 76(2):1133-1144 [PubMed: 8871226]

Vercher JL, Quaccia D, Gauthier G (1995) Oculo-manual coordination control: respective role of visual and non-visual information in ocular tracking of self-moved targets. Exp Brain Res 103(2): 311-322 [PubMed: 7789438]

Verghese P, Tyson TL, Ghahghaei S, Fletcher DC (2016) Depth perception and grasp in central field loss. Investig Ophthalmol Vis Sci 57(3):1476 [PubMed: 27031841]

von Noorden GK, Mackensen G (1962) Pursuit movements of normal and amblyopic eyes: an electroophthalmographic study 1. Physiology of pursuit movements. Am J Ophthalmol 53(2):325-336 [PubMed: 13926697]

Wann JP, Rushton S, Mon-Williams M (1995) Natural problems for stereoscopic depth perception in virtual environments. Vis Res 35(19):2731-2736 [PubMed: 7483313]

Ward MK, Bolding MS, Schultz KP, Gamlin PD (2015) Mapping the macaque superior temporal sulcus: functional delineation of vergence and version eye-movement-related activity. J Neurosci 35(19):7428-7442 [PubMed: 25972171]

Ware C, Balakrishnan R (1994) Reaching for objects in vr displays: lag and frame rate. ACM Trans Comput Hum Interact 1(4):331-356

Weichert F, Bachmann D, Rudak B, Fisseler D (2013) Analysis of the accuracy and robustness of the leap motion controller. Sensors 13(5):6380-6393 [PubMed: 23673678]

Westheimer G, Blair SM (1974) Functional organization of primate oculomotor system revealed by cerebellectomy. Exp Brain Res 21(5):463-472 [PubMed: 4374369]

Wright KW, Spiegel PH, Thompson LS (2006) Handbook of pediatric strabismus and amblyopia Springer, Berlin

Yang Q, Bucci MP, Kapoula Z (2002) The latency of saccades, vergence, and combined eye movements in children and in adults. Investig Ophthalmol Vis Sci 43(9):2939 [PubMed: 12202513] 


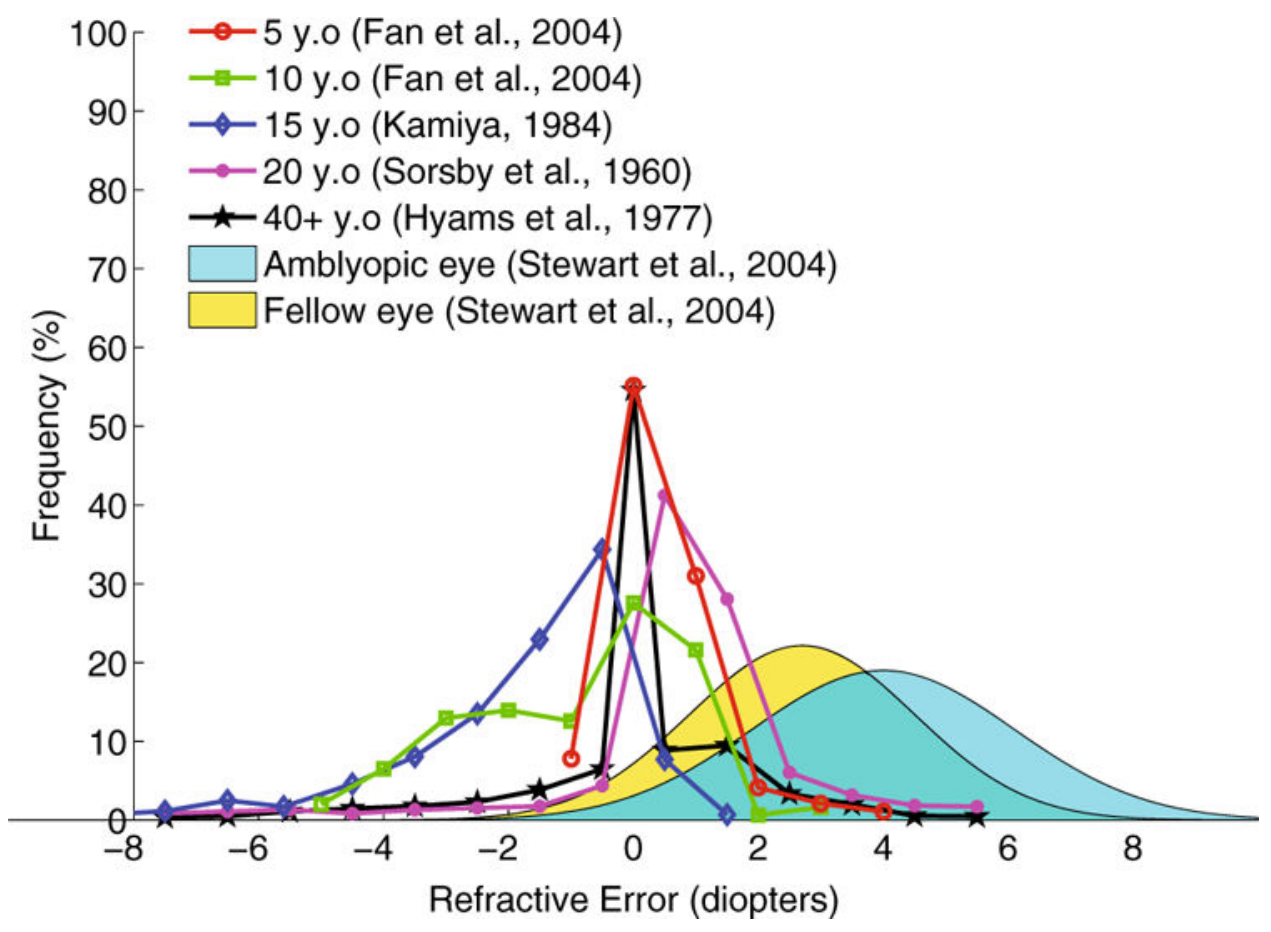

Fig. 1.

Refractive error distributions. Distributions of refractive errors for different age groups and for amblyopic children, replotted from a sample of the literature 
(a) Blur = 0 arcmin

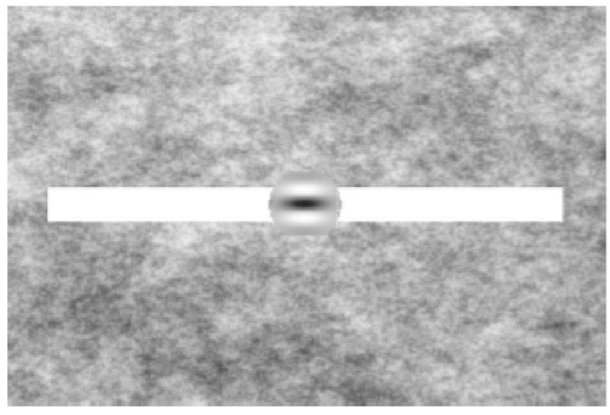

(b) Blur $=25$ arcmin

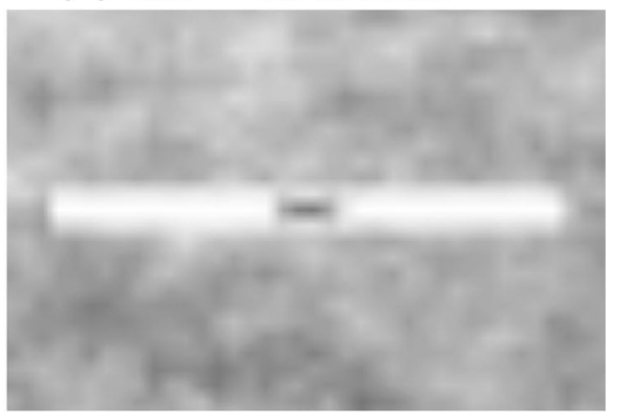

Fig. 2.

Example stimuli. The stimulus for target tracking was a Gabor patch moving on top of a white bar. The background was $1 / f$ pink noise. Left panel (a) is an example stimulus frame in the 0 arcmin blur condition. Right panel (b) is an example stimulus frame in the 25 arcmin blur condition 


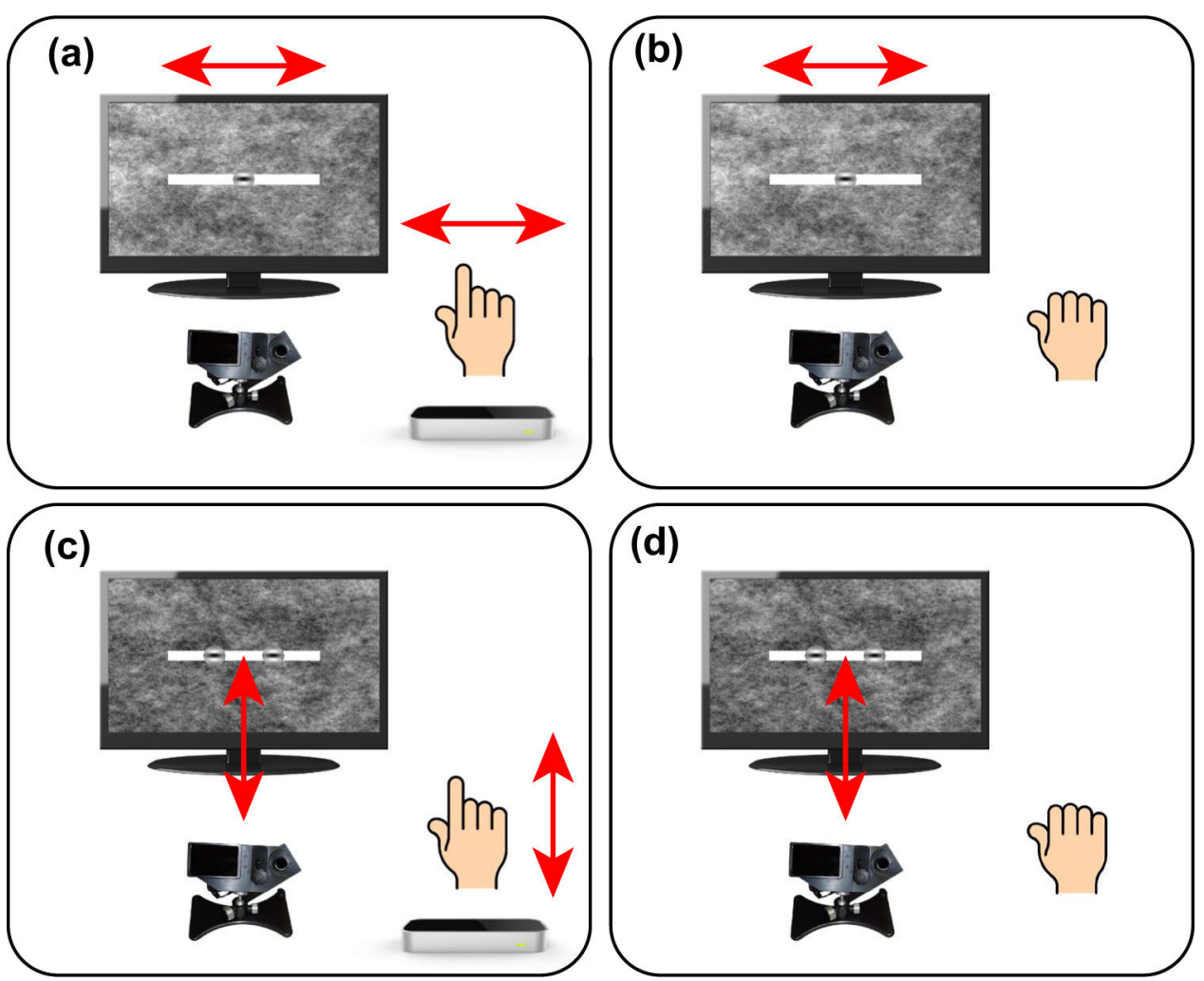

Fig. 3.

Schematics of the four experimental sessions. In all four sessions, observers' binocular gaze point was monitored at $1000 \mathrm{~Hz}$ with an Eyelink II eyetracker. a In session 1, observers smoothly moved their right index finger from right to left and back, directly above the leap motion controller. The on-screen Gabor target moved with the observers' finger. $\mathbf{b}$ In session 2, observers' hands were motionless and the Gabor target moved right to left and back, replaying one of the finger movements executed in session 1. $\mathbf{c}$ In session 3, observers smoothly moved their right index finger backward and forward, directly above the leap motion controller. The on-screen Gabor target moved in stereoscopic depth with the observers' finger. d In session 4, observers' hands were motionless and the Gabor target moved backward and forward, replaying one of the finger movements executed in session 3 


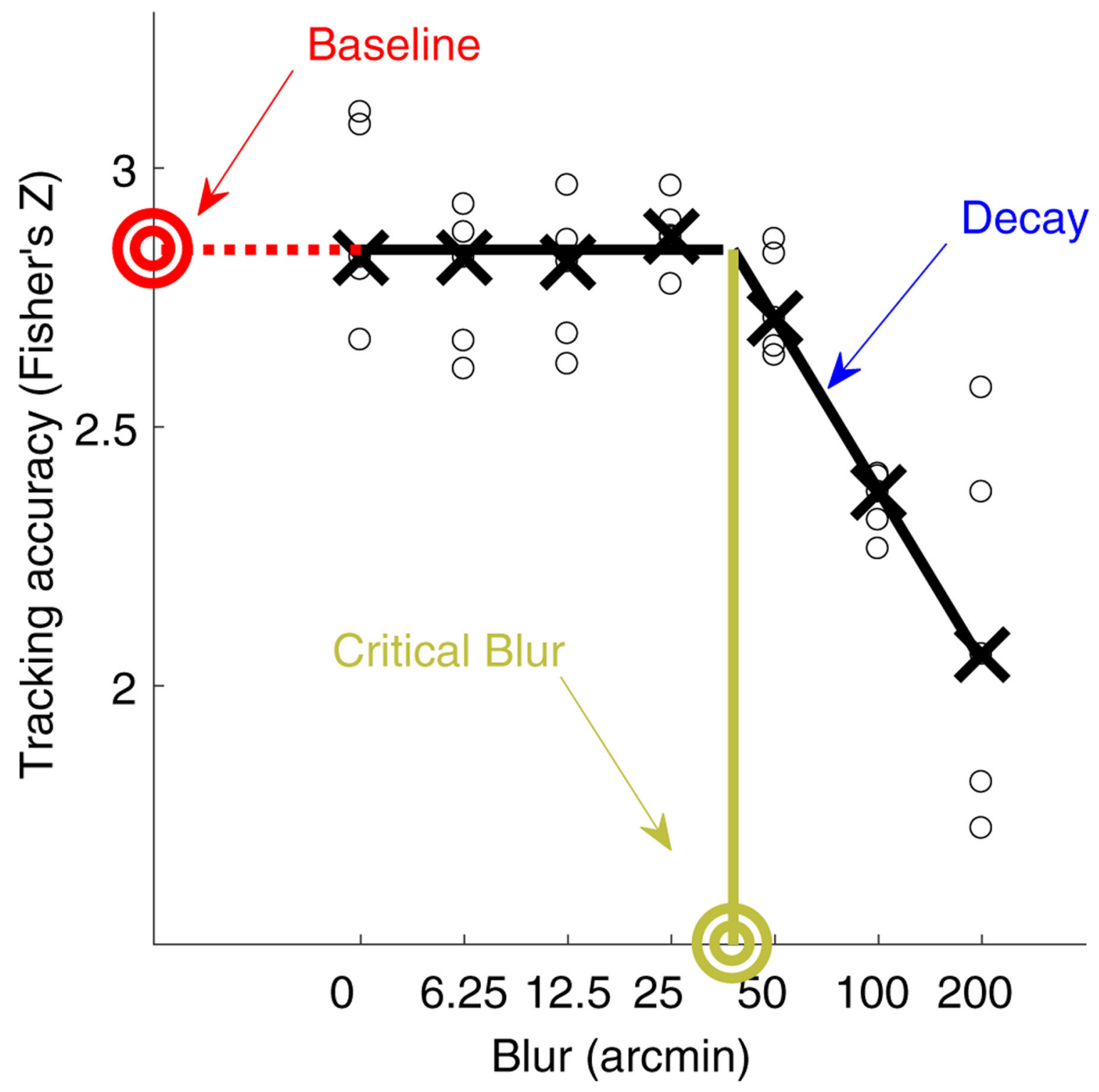

Fig. 4.

Example of individual tracking data fitted with a hinged-line function. Tracking accuracy as a function of blur for a representative observer in the binocular blur, finger tracking, horizontal pursuit condition. Black circles are individual trial accuracy data. Black crosses are medians. Black lines are the fitted hinged-line linear-log function. The $y$-axis intercept is the baseline level of accuracy (red bullseye). Beyond the critical blur level (green bullseye), accuracy begins to falls below the baseline level of tracking. The slope of the linear falloff (indicated by the blue arrow) is the rate of decay of tracking accuracy with increasing levels of blur 

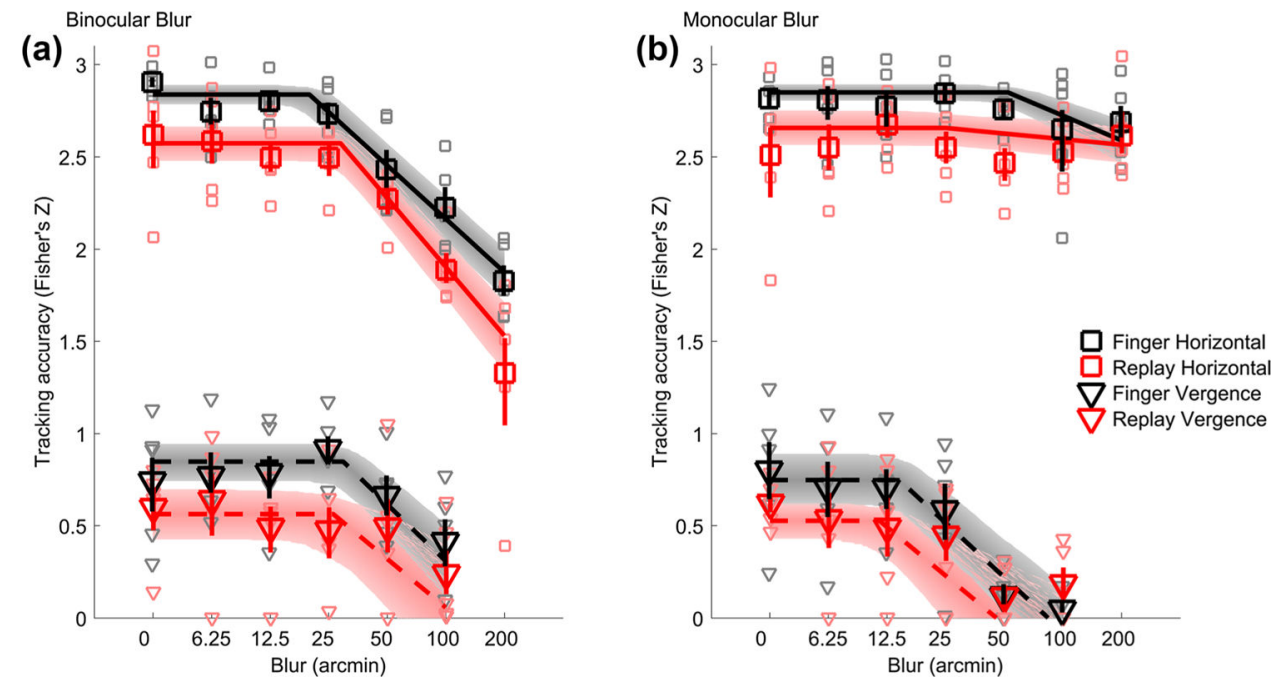

Fig. 5.

Tracking accuracy. Tracking accuracy at each blur level for binocular (a) and monocular (b) blur conditions. Data from horizontal pursuit eye movements are shown as squares and vergence pursuit eye movements as triangles. Finger tracking data are shown in black and replay tracking are in red. Large symbols represent average tracking accuracy across subjects at each blur level. Error bars are 68\% confidence intervals. Small symbols represent individual subject data. Solid and dashed lines are the average best fits of a hinged-line linearlog function for horizontal pursuit and vergence pursuit, respectively. Shaded regions are $68 \%$ confidence regions of the fitted functions. Note that the $y$-axis is scaled following Fisher's $Z$ transformation, whereas the $X$-axis is log scaled 
(a)

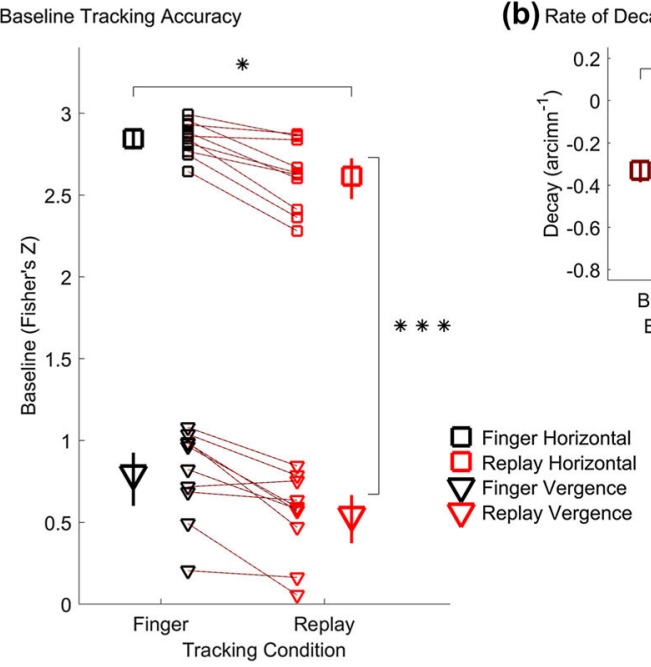

b) Rate of Decay of Tracking Accuracy
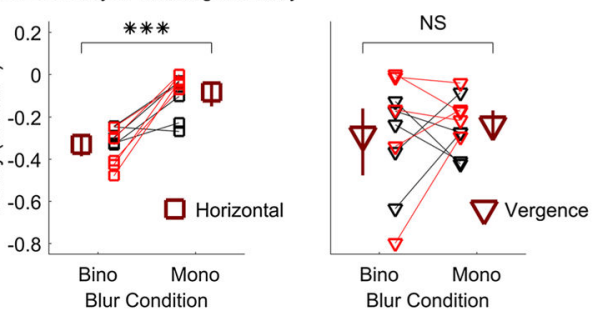

(c) Critical Refraction estimated from Critical Blur

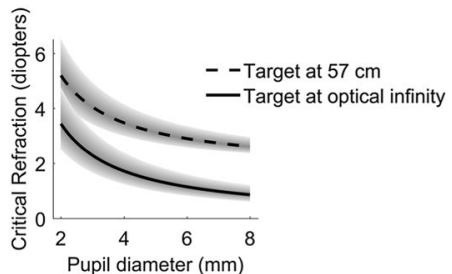

Fig. 6.

Primary findings based on the fitted parameters of the hinged-line model. Baseline level (a), rate of decay (b), and critical refraction (c) of tracking accuracy. In $\mathbf{a}, \mathbf{b}$ black symbols represent finger tracking, red symbols represent replay tracking, squares are horizontal pursuit, triangles are vergence pursuit, small symbols represent individual subject data, and dashed red-black lines connect data points belonging to individual subjects from matching blur conditions. In a large black and red symbols are mean baseline accuracy for the finger and replay conditions for both horizontal and vergence pursuit, averaged over monocular and binocular blur conditions. In $\mathbf{b}$ large brown symbols are the rate of decay of tracking accuracy for the monocular and binocular blur conditions, averaged across both finger and replay tracking conditions, for both horizontal (left) and vergence (right) pursuit. All error bars are $95 \%$ confidence intervals. ${ }^{*} p<0.05,{ }^{* * *} p<0.001$. c The estimated critical refraction (as computed from Eq. 2) at which oculomotor tracking performance may begin to decay is plotted as a function of pupil diameter for a target at $57 \mathrm{~cm}$ (dashed black curve) and for a target beyond optical infinity (continuous black curve). Shaded region encompasses the $95 \%$ confidence range of the estimate 
(a) Horizontal
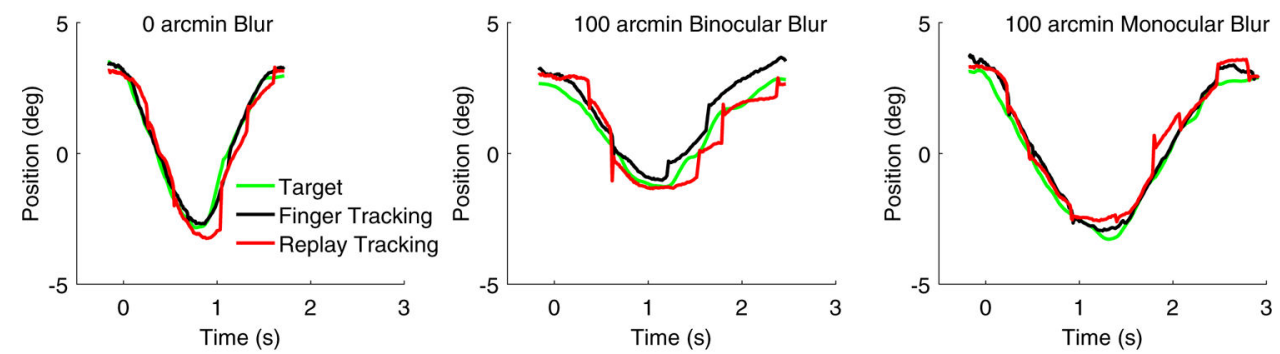

(b) Vergence
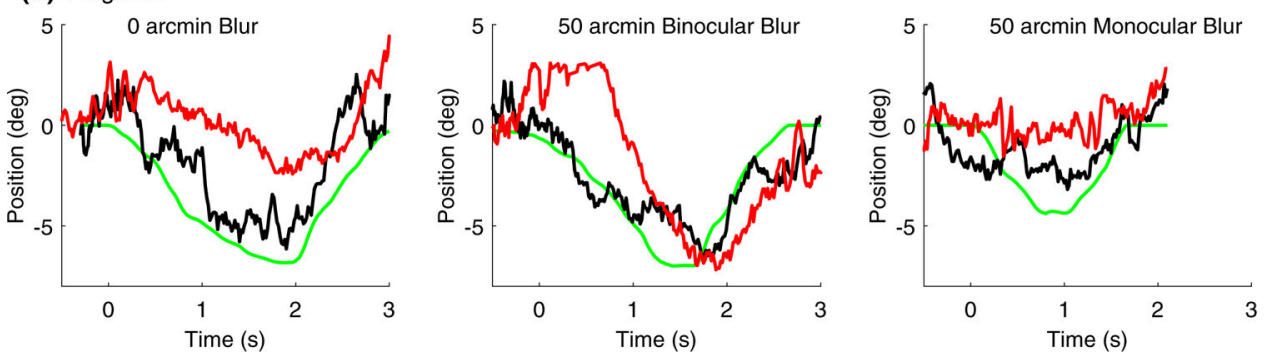

Fig. 7.

Raw tracking data from a representative observer. Target (green) and gaze position in the finger (black) and replay (red) tracking conditions are plotted as a function of time from target movement onset. a Horizontal pursuit tracking data from three trials. Left: a trial representative of the 0 arcmin blur condition. Center: a trial representative of the 100 arcmin binocular blur condition. Right: a trial representative of the 100 arcmin monocular blur condition. b Vergence pursuit tracking data from three trials. Left: a trial representative of the 0 arcmin blur condition. Center: a trial representative of the 50 arcmin binocular blur condition. Right: a trial representative of the 50 arcmin monocular blur condition 
(a) Horizontal

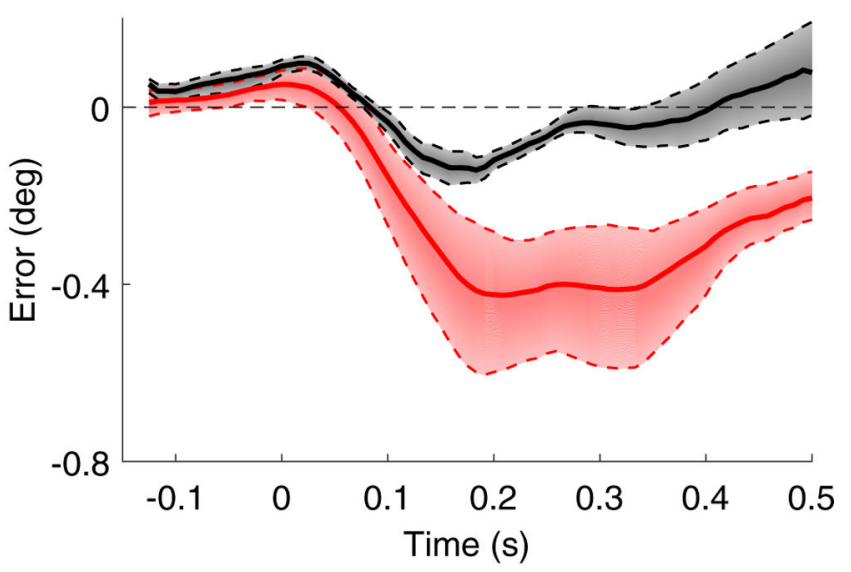

(b) Vergence

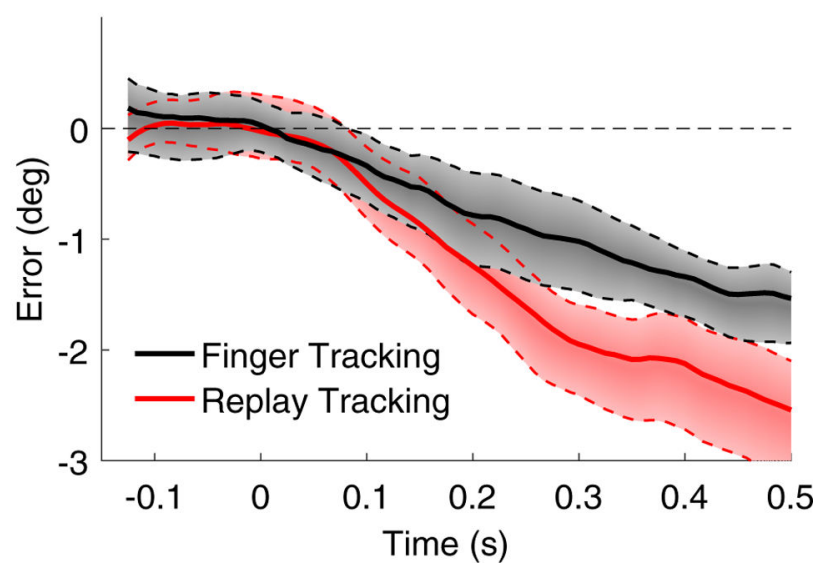

Fig. 8.

Time course of tracking error. Tracking error (the difference between gaze and finger position) is plotted as a function of time from target movement onset for a horizontal pursuit and $\mathbf{b}$ vergence pursuit tracking. Positive error values signify that the eyes were leading the target, while negative errors signify that the eyes were trailing the target. Black and red curves show the error in the finger and replay tracking conditions, respectively, averaged across all monocular and binocular blur conditions. Shaded regions bounded by dotted lines show $68 \%$ confidence intervals of the mean error trace. Note that the $y$-axes in $\mathbf{a}, \mathbf{b}$ are scaled differently 

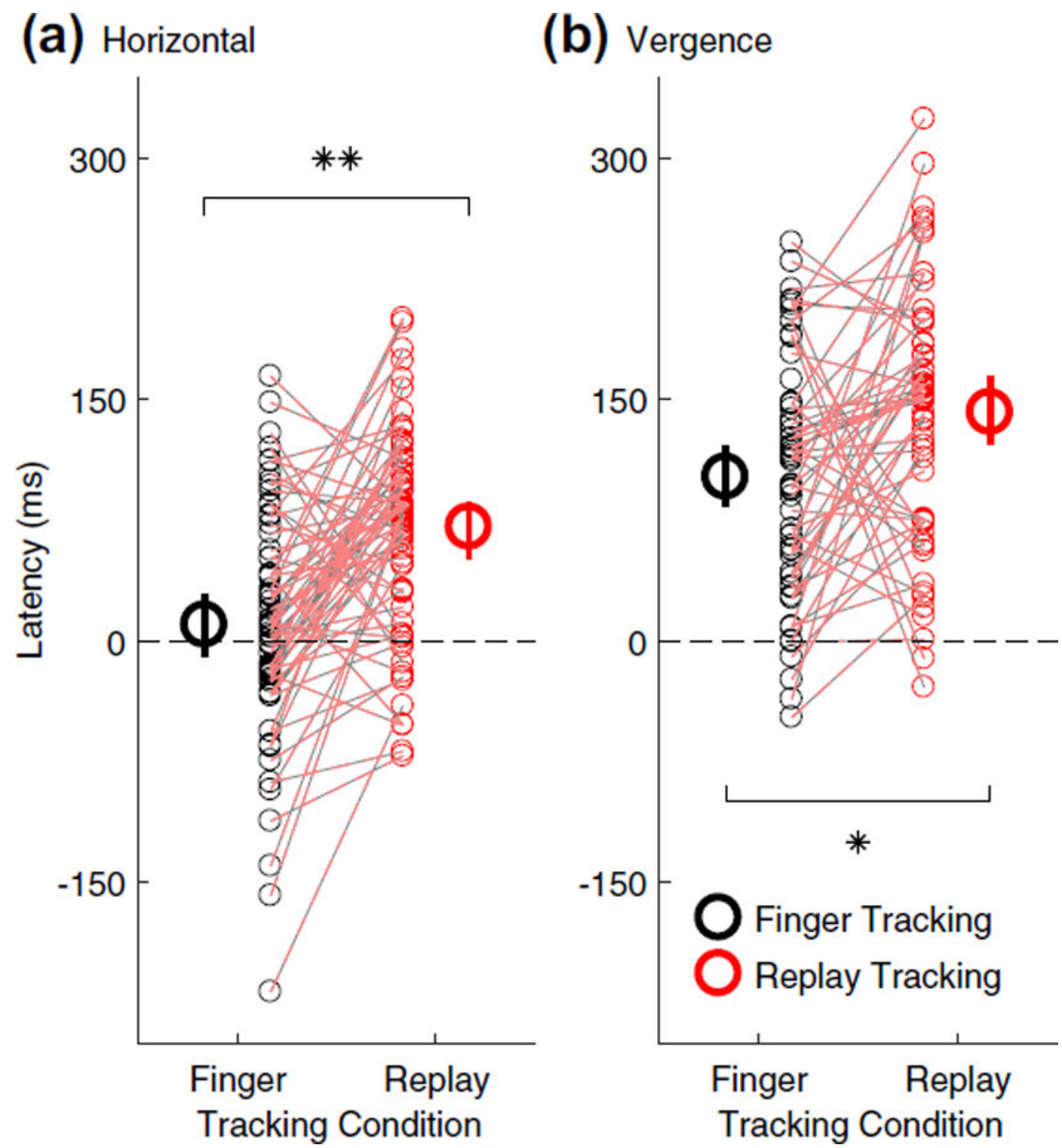

Fig. 9.

Tracking latency. Latency of gaze tracking (i.e., the difference between target and eye movement onset in milliseconds) for $\mathbf{a}$ horizontal pursuit and $\mathbf{b}$ vergence pursuit tracking. Black circles represent finger tracking, red circles represent replay tracking, small symbols represent individual subject data, and dashed red-black lines connect data points belonging to individual subjects from matching blur conditions. Large symbols are mean estimates of tracking latency in the finger and replay tracking conditions, averaged across all monocular and binocular blur conditions. Error bars are $95 \%$ confidence inter vals. Negative latencies indicate anticipatory eye movements. ${ }^{*} p<0.05, * * p<0.01$ 
(a) Horizontal

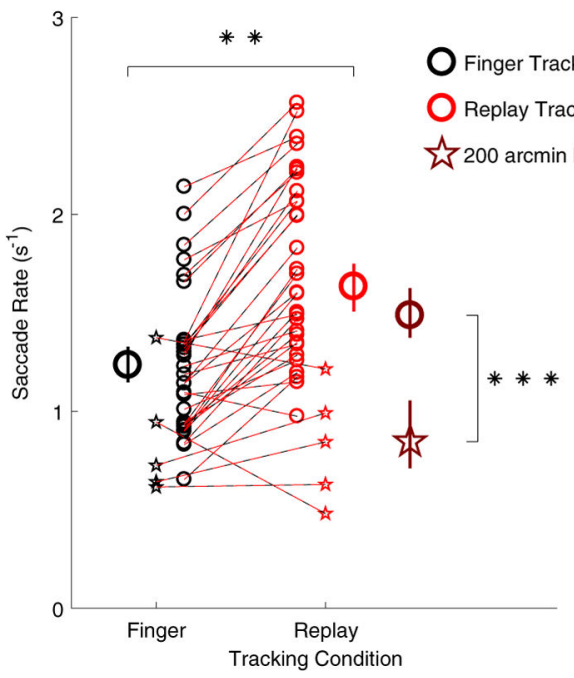

(b) Vergence

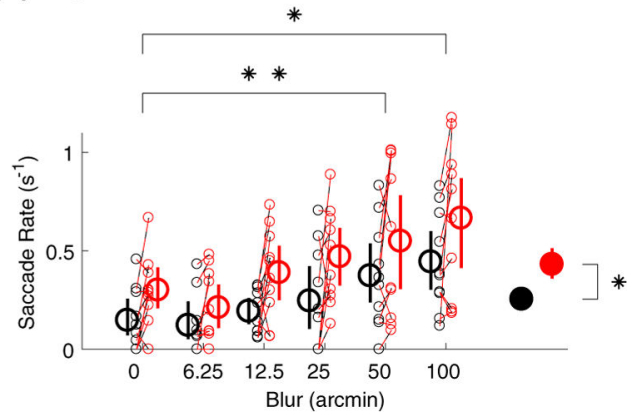

Fig. 10.

Saccade rate. Number of saccades per second occurring during (a) horizontal pursuit and (b) vergence pursuit tracking. Black symbols represent finger tracking, red symbols represent replay tracking, small symbols represent individual subject data, and dashed red-black lines connect data points belonging to individual subjects from matching blur conditions. In (a) small star symbols are data from the 200 arcmin binocular blur condition, while small circles are data from all other conditions. Large black and red circles are mean latencies for the finger and replay tracking conditions, respectively, averaged across all blur conditions. The brown star symbol represents the average saccade rate for the 200 arcmin binocular blur condition, whereas the brown circle represents the average saccade rate for all the other conditions. In (b), saccade rate during vergence tracking is plotted as a function of blur level. Large empty circles are mean saccade rate at each blur level, averaged across monocular and binocular blur conditions. Filled black and red circles are mean saccade rate during finger and replay tracking, respectively, averaged across all blur conditions. All error bars are $95 \%$ confidence intervals. $* p<0.05, * * p<0.01, * * * p<0.001$ 